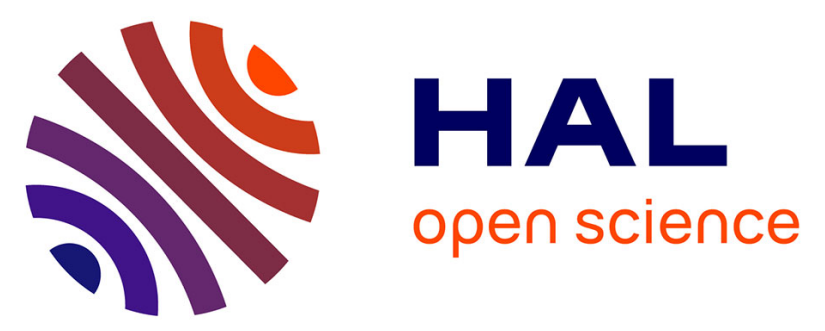

\title{
Glutamate-heme ester bond formation is disfavoured in flavocytochrome P450 BM3: Characterization of glutamate substitution mutants at the heme site of $\mathrm{P} 450$ BM3
}

Hazel M. Girvan, Colin W. Levy, Paul Williams, Karl Fisher, Myles R. Cheesman, Stephen E. J. Rigby, David Leys, Andrew W. Munro

\section{To cite this version:}

Hazel M. Girvan, Colin W. Levy, Paul Williams, Karl Fisher, Myles R. Cheesman, et al.. Glutamateheme ester bond formation is disfavoured in flavocytochrome P450 BM3: Characterization of glutamate substitution mutants at the heme site of P450 BM3. Biochemical Journal, 2010, 427 (3), pp.455-466. 10.1042/BJ20091603 . hal-00479273

\section{HAL Id: hal-00479273 https://hal.science/hal-00479273}

Submitted on 30 Apr 2010

HAL is a multi-disciplinary open access archive for the deposit and dissemination of scientific research documents, whether they are published or not. The documents may come from teaching and research institutions in France or abroad, or from public or private research centers.
L'archive ouverte pluridisciplinaire HAL, est destinée au dépôt et à la diffusion de documents scientifiques de niveau recherche, publiés ou non, émanant des établissements d'enseignement et de recherche français ou étrangers, des laboratoires publics ou privés. 


\title{
Glutamate-heme ester bond formation is disfavoured in flavocytochrome P450 BM3
}

\section{Characterization of glutamate substitution mutants at the heme site of P450 BM3}

\author{
${ }^{1}$ Hazel M. Girvan, ${ }^{1}$ Colin W. Levy, ${ }^{1}$ Paul Williams, ${ }^{1}$ Karl Fisher ${ }^{2}$ Myles R. Cheesman, \\ ${ }^{1}$ Stephen E. J. Rigby, ${ }^{1}$ David Leys, and ${ }^{1}$ Andrew W. Munro \\ ${ }^{1}$ Faculty of Life Sciences, Manchester Interdisciplinary Biocentre, University of Manchester, \\ 131 Princess Street, Manchester M1 7DN, UK. ${ }^{2}$ School of Chemical Sciences, University of \\ East Anglia, Norwich NR4 7TJ, UK.
}

the: Structure and catalysis in P450 BM3 mutants

Address correspondence to: A. W. Munro or H. M. Girvan. Tel: +44 161 3065151; Fax: +44

161 3068918; E-mail: Andrew.Munro@Manchester.ac.uk or

Hazel.Girvan@Manchester.ac.uk

Running Title: Structure and catalysis in P450 BM3 mutants 


\section{Synopsis}

Bacillus megaterium flavocytochrome P450 BM3 (BM3, CYP102A1) is a biotechnologically important cytochrome P450/P450 reductase fusion enzyme. Mutants I401E, F261E and L86E were engineered near the heme 5-methyl group, to explore ability of the glutamate carboxylates to form ester linkages to the methyl group, as observed for eukaryotic CYP4 relatives. Although no covalent linkage was detected, mutants displayed marked alterations in substrate/inhibitor affinity, with L86E and I401E mutants having lower $K_{\mathrm{d}}$ values for arachidonic acid and lauric acid than wild-type (WT) BM3. All mutations induced positive shifts in heme $\mathrm{Fe}$ (III)/Fe(II) potential, with substrate-free I401E $(-219 \mathrm{mV})$ being $>170 \mathrm{mV}$ more positive than WT BM3. The elevated potential stimulated FMN-to-heme electron transfer $\sim 2$-fold (to $473 \mathrm{~s}^{-1}$ ) in I401E, and resulted in stabilization of $\mathrm{Fe}(\mathrm{II}) \mathrm{O}_{2}$ complexes in the I401E/L86E P450s. EPR demonstrated some iron coordination by glutamate carboxylate in L86E/F261E mutants, indicating structural plasticity in the heme domains. The $\mathrm{Fe}(\mathrm{II}) \mathrm{O}_{2}$ complex is EPR silent, likely resulting from antiferromagnetic coupling between $\mathrm{Fe}(\mathrm{III})$ and bound superoxide in a ferric superoxo species. Structural analysis of mutant heme domains revealed modest rearrangements, including altered heme propionate interactions that may underlie thermodynamic perturbations observed. The mutant flavocytochromes demonstrated WT-like hydroxylation of lauric acid, but regioselectivity was skewed towards $\omega-3$ hydroxylaurate formation in F261E and towards $\omega-1$ hydroxylaurate production in I401E. Our data point strongly to a likelihood that Glu-heme linkages are disfavoured in this most catalytically efficient P450, possibly due to the absence of a methylene radical species during catalysis.

Keywords: cytochrome P450, electron paramagnetic resonance, ferrous-oxy complex, electron transfer, substrate oxidation, potentiometry.

Abbreviations: BM3 - Bacillus megaterium flavocytochrome P450 BM3; CN - sodium cyanide; CYP - cytochrome P450; KPi - potassium phosphate; NPG - N-palmitoylglycine; PDA photodiode array; PEG - polyethylene glycol; P450 - cytochrome P450; WT - wild-type; 4-PIM - 4-phenylimidazole. 


\section{Introduction}

The cytochromes P450 (P450s) are a diverse enzyme superfamily, most of whose members catalyse reductive scission of molecular oxygen and the introduction of an oxygen atom into an array of organic substrates [1]. The 57 human P450s are involved in e.g. xenobiotic and prescribed drug metabolism, steroid and lipid metabolism [2], while bacterial isoforms have roles in e.g. synthesis of polyketides, biotin biogenesis, degradation of organic molecules for energy and modification of various lipids [3-6]. Activation of molecular oxygen is achieved at a $b$-type heme iron that is proximally coordinated to the protein via a cysteine ligand in the thiolate form [7]. The $\mathrm{P} 450$ heme iron is ferric in the resting state, but is reduced to ferrous by a redox partner (usually containing iron-sulfur or flavin redox cofactors) [8]. Oxygen binds to ferrous iron and further reduction/protonation of the ferrous-oxy complex leads to the formation of reactive intermediates, and ultimately to a ferryl-oxo species that catalyses oxygen insertion into a substrate bound in the $\mathrm{P} 450$ active site [1,9].

While $c$-type heme macrocycles are covalently linked to their apoproteins by thioether bonds to heme vinyl groups, involving (usually) cysteine side chains, most $b$-type hemes are not covalently attached to the protein by linkages other than axial ligands to the heme iron [10]. This was thought to be exclusively the case for the P450s until studies revealed covalent binding of the heme macrocycle in mammalian members of the CYP4 family [11,12]. These bonds formed through turnover-dependent linkage of a conserved acidic residue (a glutamate) with a heme methyl group [13]. The possibility of stabilizing heme binding (or avoiding perturbing heme iron thiolate coordination and inducing inactive P420 formation) in other P450s was investigated by rationally engineering a glutamate residue close to a heme methyl in the Pseudomonas putida P450cam (CYP101A1) camphor hydroxylase and the Bacillus megaterium P450 BM3 (CYP102A1) fatty acid hydroxylase [14,15]. P450 BM3 (BM3) is more closely structurally/functionally related to eukaryotic CYP4s than is P450cam. It is a soluble enzyme with its redox partner (cytochrome P450 reductase or CPR) fused to the P450 in a single polypeptide, and has potential biotechnological applications [6]. For the G248E P450cam mutant, extensive turnover with camphor produced $\sim 40 \%$ covalent ligation of the heme group, but inactivated the enzyme [14]. No significant amount of covalent binding of the heme group occurred for the BM3 A264E mutant [15]. However, the BM3 A264E mutant instead formed a distal glutamate ligation to the heme iron [15]. While this finding was exploited to generate several other novel heme ligand sets through further mutagenesis (e.g. Lys/Met/His distal ligands in A264K/M/H mutants) [16,17], it remained unclear whether linkages between the BM3 heme 5methyl group and P450 glutamate residues could be formed if glutamates were engineered at alternative positions in the heme binding pocket. To investigate further, we engineered glutamate residues as close as possible to the heme 5-methyl group with minimal disruption to protein structure and function. Certain residues close to heme methyls (e.g. Thr268, Phe393 and Cys400) were not selected for mutation, as variants at these positions would perturb function by affecting catalytic proton relay (Thr268), regulation of heme iron potential and reactivity (Phe393) and proximal ligation of the heme iron (Cys400) [18-20]. Residues Leu86, Phe261, and Ile401 were found to fulfill the proximity criteria, and there was not other evidence for their essentiality for e.g. folding or catalysis. Indeed, recent studies of a BM3 I401P mutant indicated that this enzyme had enhanced affinity $\left(K_{\mathrm{m}}\right)$ for and catalytic activity $\left(k_{\mathrm{cat}}\right)$ towards the lipid substrate lauric acid [21].

In this paper we report spectroscopic, structural and thermodynamic properties of the L86E, F261E and I401E mutants of the intact flavocytochrome P450 BM3 enzyme (where the P450 is fused to its FAD- and FMN-containing CPR partner) and of its heme (P450) domain. Each mutation was generated due to predicted proximity of the introduced glutamate residue to the heme 5-methyl group, and in view of their distinctive predicted orientations towards the methyl 
group. The results demonstrate ineffective covalent modification of the heme macrocycle in these BM3 mutants by comparison with the previously studied mammalian CYP4s. However, the mutations at distinct positions around the BM3 heme have major effects on thermodynamic and catalytic properties of BM3, and provide important information on conformational regulation and on determinants of stabilization of the ferrous iron oxygen complex.

\section{Experimental}

Site directed mutagenesis. Point mutants F261E, L86E and I401E were made in both the heme domain and intact flavocytochrome plasmid constructs (pBM20 and pBM25, respectively [22,23]), using the Stratagene QuikChange mutagenesis system. Oligonucleotide primers L86EFor (CAGGAGACGGGGAATTCACAAGCT) and L86ERev (AGCTTGTGAATTCCCCGTCTCCTG) were used to generate the L86E mutants. The mutated codon is in bold and an EcoRI restriction site added by silent mutation is underlined. Primers F261EFor (CGCTATCAAATTATTCACGAATTAATTGCGGG) and F261ERev (CCCGCAATTAATTCTGTAATAATTTGATAGCG) were used for F261E mutants (mutated codon in bold). I401E mutants were made using primers I401EFor (CGGTCAGAGGGCCTGTGAAGGTCAGCAGTTCGC) I401ERev (GCGAACTGCTGACCTTCACAGGCCCTCTGACCG) which contain an Eco0109 I restriction enzyme site introduced by silent mutations, shown underlined (mutated codon in bold).

Protein expression and purification. Both wild-type (WT) and mutant heme domain and flavocytochrome constructs were expressed in E. coli TG1 cells. Cells were harvested and lysed as described previously [16,23]. Purification was by ammonium sulfate precipitation, DEAE and Q-Sepharose anion exchange and hydroxyapatite chromatography, with a final Sephacryl 200 gel filtration stage to obtain a high level of purity for crystallography, as described elsewhere [16,17]. After each stage of purification the purity was checked by determining the total P450 heme $\left(\mathrm{A}_{418}\right)$ to $\left(\mathrm{A}_{280}\right)$ protein to ratio, or Rz (Reinheitzahl) value. At each stage, the most pure fractions by this criterion were pooled and taken forward. Purity was verified by SDS-PAGE and by Rz values of $>1.7$ and $>0.9$ for heme domain and flavocytochrome P450 BM3 enzymes, respectively.

Assessment of covalent heme macrocycle ligation. Covalent ligation of the heme macrocycle to the protein was investigated by heme staining on SDS-PAGE. P450 samples were resolved on $12 \%$ and $8 \%$ gels for heme domain and flavocytochrome enzymes, respectively. Samples were incubated (for 45 minutes) in the presence of (i) substrate (arachidonate, $1 \mathrm{mM}$ ), (ii) sodium dithionite (10-fold molar excess), (iii) substrate together with sodium dithionite, (iv) substrate and NADPH $(2 \mathrm{mM})$, (v) hydrogen peroxide $\left(\mathrm{H}_{2} \mathrm{O}_{2}, 0.67 \%\right.$ v/v) or $(\mathrm{vi}) \mathrm{H}_{2} \mathrm{O}_{2}$ together with substrate, prior to electrophoresis. A positive control of horse heart cytochrome $c$ was included on each gel. Following heme staining [15], all gels were also stained with coomassie blue to check for suitable quantities of protein and positions of proteins on the gel.

Spectroscopic analysis. UV-visible spectroscopy. All UV-visible absorption spectra were collected on a Cary 50 scanning spectrophotometer (Varian) using a $1 \mathrm{~cm}$ path length quartz cuvette. Concentrations of purified proteins were calculated by the method of Omura and Sato [24] using an extinction coefficient of $\Delta \varepsilon_{450-490}=91 \mathrm{mM}^{-1} \mathrm{~cm}^{-1}$ from the difference spectrum generated by subtracting the absolute spectrum of the reduced $\mathrm{P} 450$ from that for the reduced CObound species form. This process also verified that cysteine thiolate coordination was maintained in each mutant. $\mathrm{P} 450$ concentrations were also verified using the coefficients $\varepsilon_{418}=95 \mathrm{mM}^{-1} \mathrm{~cm}^{-1}$ for the heme domains and $\varepsilon_{418}=105 \mathrm{mM}^{-1} \mathrm{~cm}^{-1}$ for the intact flavocytochromes [23]. 
Substrate and inhibitor binding analysis. Determination of the binding constants ( $K_{\mathrm{d}}$ values) for the substrates arachidonic acid and lauric acid, and for the inhibitors 4-phenylimidazole (4-PIM) and sodium cyanide $(\mathrm{CN})$ was done by optical titration of substrates/ligands against the WT and mutant BM3 heme domains, as described [16,23]. $K_{\mathrm{d}}$ values were determined from plots of induced absorption changes versus ligand concentration, with data fitted using a standard hyperbolic function or (where the $K_{\mathrm{d}}$ value was not $\geq 5 \mathrm{x}$ the $\mathrm{P} 450$ concentration) to a quadratic function designed for tight-binding ligands, as described previously [25]. Data were fitted using Origin software (OriginLab, Northampton, MA).

$$
A_{o b s}=\left(A_{\max } / 2 E t\right) \times\left(S+E t+K_{d}\right)-\left(\left(\left(S+E t+K_{d}\right)^{2}-(4 \times S \times E t)\right)^{0.5}\right) \quad \text { Equation } 1
$$

In Equation 1, Aobs is the observed absorbance change at substrate/ligand concentration $S, A$ max is the absorbance change at substrate/ligand saturation, Et is the P450 BM3 enzyme concentration and $K_{d}$ is the dissociation constant for the P450 BM3-substrate/ligand complex.

EPR spectroscopy. EPR spectra for ferric samples of the WT and L86E, F261E and I401E heme domains were collected using an EPR spectrometer comprising an ER200D electromagnet and microwave bridge interfaced to a EMX control system (Bruker Spectrospin), and fitted with a liquid helium flow cryostat (ESR-9, Oxford Instruments) and a dual-mode X-band cavity (Bruker type ER4116DM). Spectra were collected for WT and mutant heme domains $(\sim 200 \mu \mathrm{M})$ in assay buffer $(100 \mathrm{mM}$ potassium phosphate, $\mathrm{pH} 7.0)$ at $10 \mathrm{~K}$, with a microwave power of $2.08 \mathrm{~mW}$ and a modulation amplitude of $10 \mathrm{G}$. To obtain EPR spectra of oxyferrous forms of WT and mutant BM3 heme domains, proteins were reduced anaerobically by addition of a stoichiometric amount of sodium dithionite. P450 reduction was verified spectrophotometrically, and samples were then transferred directly to an EPR tube. A few bubbles of air were introduced into samples, which were then either frozen immediately using liquid nitrogen, or after a one minute incubation. EPR spectra of the oxyferrous samples were obtained using a Bruker E500 ELEXSYS instrument operating at X-band. Temperature was controlled using an Oxford Instruments ESR900 liquid helium cryostat together with a ITC503 temperature controller. EPR spectra were obtained at 10 $\mathrm{K}$ using $0.5 \mathrm{~mW}$ microwave power, a modulation frequency of $100 \mathrm{KHz}$, and a modulation amplitude of 5 G. EPR spectra of samples of ferric WT/mutant BM3 heme domains were also collected at the same protein concentration as for the oxyferrous samples.

Resonance Raman spectroscopy. Resonance Raman (RR) spectra were collected using a $15 \mathrm{~mW}$ $406.7 \mathrm{~nm}$ radiation source at the sample delivered from a Coherent Innova 300 krypton ion laser, with spectral acquisition by a Renishaw micro-Raman system 1000 spectrometer. Spectra of WT and mutant heme domains $(50 \mu \mathrm{M})$ were collected in both the presence and absence of arachidonate at ambient temperature, and extended scans obtained from $200-1700 \mathrm{~cm}^{-1}$. Samples were held in a capillary under a microscope and each was subjected to $5 \times 15 \mathrm{~s}$ exposures. Data processing, curve fitting, and band assignment was done using GRAMS/32 software (Thermo Scientific).

Steady-state kinetic and product characterization studies. Steady-state measurements of the substrate-dependent (lauric acid and arachidonic acid) NADPH oxidation were made for the mutant flavocytochromes, as described previously [16,23]. Products of lauric acid oxidation were investigated for WT/mutant flavocytochromes by incubating lauric acid $(800 \mu \mathrm{M})$ with NADPH $(2 \mathrm{mM})$ and $0.5 \mu \mathrm{M}$ enzyme for 1 hour at $25^{\circ} \mathrm{C}$. Reactions were halted by addition of $\mathrm{HCl}$ to acidify the mixtures to $\mathrm{pH} \sim 2$. Lauric acid substrate and hydroxylated products were isolated from the mixtures by binding to SPE columns (Phenomenex, Macclesfield UK) and with elution using methanol. A small amount of phenylacetic acid dissolved in methanol $(20 \mu \mathrm{L}$ of a $70 \mu \mathrm{M}$ 
stock), which was used as an internal standard, was added to the isolated lipids before evaporating them to dryness. The residue was reconstituted with $150 \mu \mathrm{L} N, O$ Bis(trimethylsilyl)trifluoroacetamide (BSTFA), $450 \mu \mathrm{L}$ Trimethylchlorosilane (TMCS) was added and the mixture was incubated at $60^{\circ} \mathrm{C}$ for $60 \mathrm{~min}$. An aliquot $(1 \mu \mathrm{L})$ of the derivatized extract was analyzed by GC-MS. Quantitative analyses were performed on a Finnigan Polaris $Q$ GC/MS ${ }^{n}$ Benchtop Ion Trap Mass Spectrometer fitted with a $30 \mathrm{~m}$ Phenomenex ZB5-MS column (ID $0.32 \mathrm{~mm}$ : phase thickness $320 \mathrm{~nm}$ ) and a liquid autosampler. High purity helium was used as the carrier gas at a flow rate of $1.5 \mathrm{~mL} / \mathrm{min}$ in constant flow mode. Samples were injected into a Progammable Temperature Vapouriser (PTV) in Constant Temperature (CT) mode with a split ratio of $50: 1$, and the injection port maintained at $250^{\circ} \mathrm{C}$. The Gas Chromatograph to Mass spectrometer interface temperature was $240^{\circ} \mathrm{C}$ and the Ion trap temperature was maintained at $100^{\circ} \mathrm{C}$ throughout. The initial oven temperature was $80^{\circ} \mathrm{C}$, held for two minutes after sample injection, programmed to $275^{\circ} \mathrm{C}$ at $15^{\circ} \mathrm{C} / \mathrm{min}$, and held for $4 \mathrm{~min}$. The mass spectrometer scanned from 45-450 Daltons at 3 scans/second in $\mathrm{MS}^{1}$ mode. Retention times for $\omega-1, \omega-2$ and $\omega-3$ hydroxylaurate products were $13.27,13.16$ and 12.97 minutes, respectively.

Potentiometric analysis. Spectroelectrochemical titrations to determine heme iron redox potential $\left(\mathrm{Fe}^{3+} / \mathrm{Fe}^{2+}\right.$ couple) were done as described previously [19,26,27]. Measurements were made for $\mathrm{WT} /$ mutant heme domains at a concentration of $6-10 \mu \mathrm{M}$, both in the presence and absence of saturating arachidonate $(\mathrm{ca} 100 \mu \mathrm{M})$, in redox buffer $(100 \mathrm{mM}$ potassium phosphate $(\mathrm{KPi}), \mathrm{pH}$ $7.0,10 \%$ glycerol) at $25^{\circ} \mathrm{C}$. All measurements were made in a Belle technology glove box under a nitrogen atmosphere, and with oxygen concentration maintained at less than $2 \mathrm{ppm}$. Spectra were collected on a Cary 50 scanning spectrophotometer (Varian) external to the glove box, with a fibre-optic UV-visible probe running from the spectrophotometer to the enzyme sample in the anaerobic box. Potentials were measured with a Thermo Russell calomel electrode attached to a Hanna pH211 microprocessor meter.

Crystallography. Each BM3 heme domain mutant was crystallized by the sitting drop technique at $4^{\circ} \mathrm{C}$. Drops were prepared by addition of $2 \mu \mathrm{l}$ of $12 \mathrm{mg} / \mathrm{ml}$ mother liquor to $2 \mu \mathrm{l} 12 \mathrm{mg} / \mathrm{ml}$ heme domain in $10 \mathrm{mM}$ Tris. $\mathrm{HCl} \mathrm{pH} 7.4$ (plus or minus $500 \mu \mathrm{M} \mathrm{N}$-palmitolylglycine [NPG]). I401E mutant crystals were obtained with mother liquor containing $100 \mathrm{mM}$ cacodylic acid, $\mathrm{pH}$ 6.0, containing $18 \%$ PEG 3350 and $140 \mathrm{mM} \mathrm{MgCl}_{2}$. Mother liquor containing $100 \mathrm{mM}$ cacodylic acid, pH 6.0, 13\% PEG 3350, and $140 \mathrm{mM} \mathrm{MgCl}_{2}$ was used to obtain F261E crystals. L86E crystals were obtained with mother liquor containing $100 \mathrm{mM}$ cacodylic acid, $\mathrm{pH} 6.0$, $16 \%$ PEG 3350, and $140 \mathrm{mM} \mathrm{MgCl}$. Crystals were flash frozen in liquid nitrogen using $10 \%$ PEG 200 as cryoprotectant. Data for all crystal structures were collected to respective resolutions from a single cryofrozen crystal at ESRF Grenoble, France or Diamond, Harwell, UK beamlines. The data were scaled and integrated using the XDS package [28] and subsequently handled using the CCP4 suite [29]. All structures were solved using difference Fourier methods. Refinement and model building were carried out using Refmac 5 [30] and COOT [31]. Data and final refinement statistics are in Supplementary data Table 1.

Stopped-flow kinetic studies. Stopped-flow absorption measurements were made using an Applied Photophysics SX18 MVR stopped-flow spectrophotometer in an anaerobic environment (Belle Technology glove box) to maintain oxygen levels at $<2 \mathrm{ppm}$. Both single wavelength and multiple wavelength (spectral acquisition) data were collected using a photodiode array (PDA) detector and XSCAN software, with the first spectrum recorded $1.28 \mathrm{~ms}$ after mixing. P450 BM3 flavin (FMN)-to-heme electron transfer rates were measured for the WT and mutant flavocytochromes at $25^{\circ} \mathrm{C}$ by monitoring formation of the ferrous-CO complex at $450 \mathrm{~nm}$. All experiments were done with anaerobic assay buffer pre-saturated by extensive bubbling with $\mathrm{CO}$ gas $(\sim 975 \mu \mathrm{M})$. Reactions were initiated by mixing a $200 \mu \mathrm{M}$ NADPH solution with mutant and 
WT flavocytochromes $(3 \mu \mathrm{M})$ containing varying concentrations of arachidonic acid substrate $(0-$ $500 \mu \mathrm{M})$. P450 heme oxyferrous complex formation and stability was measured at $20^{\circ} \mathrm{C}$. An anaerobic sample of mutant or wild-type heme domain was reduced by titration with dithionite prior to stopped-flow mixing with oxygenated assay buffer. PDA measurements (from 350 to 700 $\mathrm{nm}$ ) were made for periods up to 200 seconds to observe spectral evolution and decay of the complex. Reactions were performed both for the substrate-free heme domains and for the substrate-bound forms (NPG at $50 \mu \mathrm{M})$.

\section{Results}

\section{UV-visible absorption spectroscopy studies}

The heme cofactor of $\mathrm{P} 450$ s produces a distinctive spectral signature in the UV-visible region that can be highly informative with respect to the heme iron oxidation- and spin-state, as well as highlighting the presence of ligands interacting with the heme iron at its distal face, as seen in our previous studies of BM3 mutants [15-17,32]. Absorption spectra suggested that both the purified F261E and I401E enzymes (heme domains and flavocytochromes) were extensively low-spin (LS) with Soret maxima at $419 \mathrm{~nm}$ for both heme domains. However, for L86E there was a spectral feature evident at $392 \mathrm{~nm}$ (alongside the $418 \mathrm{~nm}$ LS maximum) indicating a small proportion ( $c$ a $10 \%$ ) of high-spin (HS) ferric heme iron in this heme domain. The Soret peak for WT BM3 heme domain was at $419 \mathrm{~nm}$. In solution at room temperature, there is no indication of distal heme iron coordination by the introduced glutamate residues in any of the mutant proteins (i.e. a red shifted Soret feature at $\sim 425 \mathrm{~nm}$, as seen in the A264E mutant [15]).

The binding of fatty acid substrates to BM3 leads to displacement of a water ligand as the $6^{\text {th }}$ (axial) ligand to the heme iron, conversion of heme from LS to HS, and (from crystal structures) the stabilization of a different conformational state to that seen for the substrate-free heme domain [20,32,33]. Arachidonic acid is an excellent BM3 substrate, inducing an extensive conversion to the HS state. Optical studies of the I401E heme domain indicated a near complete arachidonate-induced conversion to HS, similar to WT BM3 (Figure 1A and 1B). By contrast, the F261E/L86E heme domains exhibited less complete arachidonate-induced HS conversions $(\sim 70 \% / 65 \%$ the extent of the WT HS shift, Figure 1B). As for WT BM3, there were smaller shifts to HS with lauric acid (dodecanoic acid) as substrate than with arachidonate in all mutants. I401E gave a much lower laurate-induced conversion to HS $(\sim 50 \%)$ than did WT heme domain, while L86E gave a conversion comparable to WT, suggesting altered binding modes for the different substrate types between mutants. The $K_{\mathrm{d}}$ data (Table 1) show that arachidonate binding remains tight in all mutants, with the L86E mutant exhibiting very tight binding $\left(K_{\mathrm{d}} \sim 20 \mathrm{nM}\right)$ despite a lower extent of HS shift than seen for WT/I401E P450s. For the F261E mutant, the extent of optical change mediated by lauric acid binding was insufficient to enable accurate $K_{\mathrm{d}}$ determination. An estimate of $>1 \mathrm{mM}$ was made, but only $\sim 10 \%$ HS heme iron accumulated in the F261E heme domain at saturating levels of lauric acid. However, $K_{\mathrm{d}}$ values for lauric acid were lower for both I401E/L86E heme domains $(11.9 / 1.0 \mu \mathrm{M})$ than for WT heme domain (89 $\mu \mathrm{M}$ ), with laurate binding for the L86E mutant improved by almost 2 orders of magnitude.

Comparative studies of WT/mutant heme domains were done for binding of the heme coordinating ligands cyanide $(\mathrm{CN})$ and 4-phenylimidazole (4-PIM). WT and all mutants gave type II optical shifts to hexacoordinated forms with the exogenous ligands replacing the distal aqua ligand, and with Soret maxima shifted to $440 \mathrm{~nm}(\mathrm{CN})$ and $424 \mathrm{~nm}$ (4-PIM) (Figure 2). 4PIM bound substantially tigher than did the polar CN ion in all cases. The $K_{\mathrm{d}}$ values are shown in Table 1 . For I401E, the $K_{\mathrm{d}}$ for 4-PIM was markedly lower than for WT $(0.1 \mu \mathrm{M}$ versus $0.85 \mu \mathrm{M})$. For WT/L86E mutant heme domains, plots of induced heme optical change versus cyanide 
concentration were hyperbolic, suggesting a single, saturable binding site on the heme iron. Affinity for cyanide was $~ 9$-fold weaker in L86E than in WT BM3, likely due to the electrostatic/steric influence of the Glu86 side chain close to the heme. However, the cyanide binding plots were sigmoidal for both the F261E/I401E heme domains, and apparent $K_{\mathrm{d}}$ values (i.e. the points of inflexion of the sigmoidal fits) were $\sim 40-50 \mathrm{mM}$ in both cases (Table 1), again indicating weaker binding of $\mathrm{CN}$ than for the WT heme domain. For F261E, repulsive electrostatic interactions of the E261 carboxylate with CN may occur, but in the I401E mutant the mutation is on the heme proximal side, and thus unlikely to diminish $\mathrm{CN}$ affinity by direct electrostatic repulsion. The basis for apparent cooperative binding of $\mathrm{CN}$ to the $1401 \mathrm{E} / \mathrm{F} 261 \mathrm{E}$ mutants remains uncertain, although it is of note that the F261E mutant crystallizes in a conformational state only rarely observed previously (see Structural studies on P450 BM3 mutants section).

Reduction of the WT and mutant heme domains showed that all were converted to the ferrous form, with their Soret bands shifted to $409 \pm 1 \mathrm{~nm}$. The Soret blue shift is consistent with retention of cysteine (Cys400) thiolate as the proximal heme ligand in all cases for the ferrous hemoproteins [34,35]. Consistent with this conclusion, all ferrous proteins bound carbon monoxide (CO) to produce Soret shifts to $448 \mathrm{~nm}$ (WT), $450 \mathrm{~nm}$ (I401E), $448 \mathrm{~nm}$ (F261E) and $450 \mathrm{~nm}$ (L86E), respectively. Thus, introduction of acidic residues on proximal and distal sides of the heme did not disrupt thiolate coordination, and all $\mathrm{Fe}$ (II)CO complexes were of the characteristic P450 spectral type, with minimal amounts of $\mathrm{P} 420$ that might indicate proximal thiol ligation. An example is shown for the F261E heme domain in Figure 2.

\section{Covalent heme macrocycle ligation}

Heme staining of WT/mutant BM3 enzymes (resolved by SDS PAGE) provided no evidence for any significant extent of covalent linkage of the heme macrocycle to the protein, even after extended turnover of WT and mutant flavocytochromes in presence of arachidonic acid substrate and $\mathrm{NADPH}$, or after incubation with $\mathrm{H}_{2} \mathrm{O}_{2}$ (either plus or minus substrate).

\section{Spectroscopic analysis of P450 BM3 mutants}

EPR. Low temperature EPR studies of ferric P450s are informative in relation to the heme iron spin-state and ligation state. EPR spectra were collected for WT and all mutant heme domains. In all cases, spectra were consistent with a near-exclusively LS ferric heme (as expected for substrate-free BM3 at cryogenic temperatures). A typical P450 rhombic spectrum was observed for WT and mutant proteins. The major set of g-values was near-identical in all cases, with $\mathrm{g}_{\mathrm{x}}=$ $2.42, \mathrm{~g}_{\mathrm{y}}=2.26, \mathrm{~g}_{\mathrm{z}}=1.92$ for WT BM3, and values of 2.42/2.26/1.93 for each of the L86E, I401E and F261E mutants. The positions of these features confirm retention of heme iron cysteinate coordination in all these P450s. For the F261E mutant, small $\mathrm{g}_{\mathrm{z}}$ features at 2.63/2.52 and $\mathrm{g}_{\mathrm{x}}$ features at $1.90 / 1.84$ are consistent with the presence of alternative LS species. In the L86E mutant there are similar (and slightly more pronounced) features at 2.58/2.53/2.48 and 1.90/1.87 (Figure 3). These minor sets of g-values are similar to those we observed for the A264E mutant of P450 BM3, where there was also crystal structure evidence for distal coordination of heme iron by Glu264 [15]. Some heterogeneity in the g-values for the Glu264-ligated form of the A264E mutant were also observed, and assigned to signals that could arise from different orientations of the Glu264 ligand, ferric iron interactions with different oxygen atoms of the carboxylate, or structural influences of active site residues (e.g. Phe87). It thus appears likely that the minor sets of g-values in the L86E/F261E mutants also originate from sub-populations in which Glu86/Glu261 ligate distally to the ferric heme iron. There is not firm evidence for such coordination at ambient temperature (or in crystals), but since EPR indicates that such Glucoordinated forms are minor species, any putative red shift of the Soret band induced might not influence the optical spectra discernibly. However, given the proximity of both Glu261 and 
Glu86 to the iron on the distal face of the heme (and since there are no such novel EPR signals in the I401E mutant on the proximal face of the heme where cysteinate ligation occurs) it appears that freezing of these mutants to $10 \mathrm{~K}$ is able to capture conformational states in which glutamate ligation to heme iron occurs to a small extent. In addition to the known BM3 substrate-free and substrate-bound heme domain conformations, we have revealed crystallographically a third conformation of the BM3 heme domain (in WT and an A264H mutant) and it is thus clear that different conformational states of the enzyme are accessible in solution [16]. EPR spectra were also recorded for ferrous WT and mutant heme domains that had been reacted with oxygen, as described in the Analysis of the formation and decay of the ferrous-oxy complex section.

Resonance Raman. Resonance Raman (RR) was applied to the BM3 heme domain mutants to probe for perturbations to the electronic properties and to the geometry of the heme and its peripheral groups. RR data show that WT and all mutant heme domains have an oxidation state marker $\left(v_{4}\right)$ at $1371 \mathrm{~cm}^{-1}$, confirming the ferric state. All are predominantly LS, with the major $v_{3}$ spin-state marker band at 1500, 1499 and $1500 \mathrm{~cm}^{-1}$ for the L86E, F261E and I401E mutants [36]. WT and mutant heme domains retain ferric heme iron on substrate addition (arachidonate), but changes in heme iron spin state equilibrium occur, with increased HS heme iron in all mutants, shown by the increased intensity of the $v_{3}$ band at $\sim 1485 \mathrm{~cm}^{-1}$. The F261E mutant has a somewhat smaller $v_{11}$ band at $1568 \mathrm{~cm}^{-1}$ than WT BM3. This band reports on electronic conjugation of porphyrin and vinyl groups, and hence on the in-plane asymmetry of the heme ring [37]. It is also affected by a $6^{\text {th }}$ ligand, and thus the small amount of glutamate coordination predicted by EPR studies of the F261E heme domain are consistent with its weaker $v_{11}$ signal.

\section{Redox Potentiometry}

Despite the apparent lack of covalent bonding between the introduced glutamate side chains and the heme methyl groups (or other parts of the macrocycle), the introduction of charged residues in the heme vicinity might affect its thermodynamic and/or catalytic properties. Thus, we analysed the heme iron redox potential in all mutants, and compared these to values for the WT heme domain plus/minus substrate (arachidonate). Redox potential values for WT and mutant BM3 heme domains are shown in Table 2. All mutations increased the potential for the $\mathrm{Fe}^{3+} / \mathrm{Fe}^{2+}$ heme iron transition. In all cases, binding of arachidonic acid induced spectral changes consistent with the accumulation of HS heme iron (see UV-visible absorption spectroscopy studies), and induced further positive shifts in heme iron potential. The substrate-free F261E heme domain has the closest potential to the WT heme domain $(-367 \pm 8 \mathrm{mV}$ versus $-395 \pm 4 \mathrm{mV}$ for WT), while L86E $(-307 \pm 5 \mathrm{mV})$ and I401E $(-219 \pm 4 \mathrm{mV})$ were much more positive. Previous studies of both BM3 and P450cam showed that the near-complete conversion of heme iron spin-state to HS on substrate binding was accompanied by elevation of heme iron potential by $>100 \mathrm{mV}$. A similar extent of heme potential shift was observed for arachidonate-bound L86E (131 mV to $-176 \pm 6$ $\mathrm{mV})$ and $\mathrm{F} 261 \mathrm{E}(170 \mathrm{mV}$ to $-197 \pm 6 \mathrm{mV})$ heme domains, although the potential shift for I401E was less substantial $(53 \mathrm{mV}$ to $-166 \pm 7 \mathrm{mV})$. Figure 4A shows exemplary spectra collected during the I401E heme domain redox titration, while Figure 4B shows overlaid Nernst function fits of heme absorption (\% heme reduced) versus applied potential for both WT and I401E heme domains in their substrate-free and arachidonate-bound forms. Previous studies of BM3 F393A/H mutants that (like I401E) are located near the heme proximal ligand (Cys400) showed that these had more positive heme iron potentials than WT BM3 and stabilized the ferrous-oxy complex of the P450 [19,38]. In view of this, we analysed the transient and steady-state kinetic properties of the thermodynamically perturbed I401E, F261E and L86E flavocytochromes P450 BM3. This was done to establish effects on turnover and reductase FMN-to-P450 heme electron transfer, and also to assess capacity of these mutants to stabilize a ferrous-oxy form that is barely detectable for WT BM3 at ambient temperature. 


\section{Flavin-to-heme electron transfer in WT and mutant P450 BM3 enzymes}

Stopped-flow studies followed the formation of the dead-end Fe(II)CO complex (with absorption maximum at $\sim 448 \mathrm{~nm}$ ) to determine FMN-to-heme electron transfer kinetics [15,39]. CO binding to ferrous BM3 heme iron is substantially faster than the inter-cofactor electron transfer rate under the conditions used [40], and thus the stopped-flow method is useful for analysing the influence of altered heme redox potentials on heme reduction rate in the glutamate substitution mutants.

For the I401E mutant, the apparent limiting rate of electron transfer $\left(k_{\text {lim }}\right)$ for the arachidonatebound enzyme is $\sim 2$-fold that for WT BM3 (473 s sersus $\left.250 \mathrm{~s}^{-1}\right)$, consistent with its more positive heme potential. Substrate-free I401E is converted rapidly and completely to its Fe(II)CO complex $\left(k_{\text {lim }}=60 \mathrm{~s}^{-1}\right)$, in contrast to WT BM3 where there is negligible Fe(II)CO complex formation for substrate-free enzyme on a stopped-flow time scale. Substrate-free I401E is extensively low-spin in its resting form, but its potential $(-219 \mathrm{mV})$ is more positive than that for the arachidonate-bound form of WT BM3, indicating the importance of heme iron thermodynamics in regulating rate and extent of heme reduction. The arachidonate-bound F261E mutant exhibits only partial conversion to the HS form, and has a $k_{\mathrm{lim}}$ of $58 \mathrm{~s}^{-1}$ for $\mathrm{Fe}$ (II)CO complex formation, indicating substantially slower FMN-to-heme electron transfer compared to WT BM3. There is much less Fe(II)CO complex formation in the substrate-free F261E enzyme $(\sim 20 \%)$ with a $k_{\lim }$ of $27 \mathrm{~s}^{-1}$. For the L86E mutant (that has a more positive potential than WT BM3 in both substrate-free and arachidonate-bound forms, and some HS content in the ferric, substrate-free enzyme), the $k_{\lim }$ for $\mathrm{Fe}(\mathrm{II}) \mathrm{CO}$ formation is $213 \mathrm{~s}^{-1}$ in the arachidonate-bound form and $102 \mathrm{~s}^{-1}$ in the substrate-free form.

These data are consistent with a major role for heme potential in regulating FMN-to-heme electron transfer. In the I401E/L86E mutants, the potential of the substrate-free heme iron (-219 $\mathrm{mV} /-307 \mathrm{mV}$, respectively) is much more positive than for the substrate-free WT BM3, and this is certainly a factor underlying the efficient FMN-to-heme electron transport in these enzymes. There is extensive formation of the Fe(II)CO complex in the substrate-free forms of I401E/L86E, whereas this does not occur to any considerable extent for WT BM3 (or the F261E mutant). The midpoint reduction potential of the (catalytically relevant) anionic semiquinone form of BM3's FMN cofactor (the oxidized/semiquinone couple) is $-240 \pm 10 \mathrm{mV}$, while that for the semiquinone/hydroquinone couple is $-160 \pm 10 \mathrm{mV}$ at $\mathrm{pH} 7.0$ [41]. For each of the mutants generated here, FMN-to-heme electron transfer is strongly thermodynamically favoured from the FMN semiquinone to the substrate-bound ferric heme iron.

\section{Analysis of the formation and decay of the ferrous-oxy complex}

The more positive redox potential of the I401E and L86E mutants suggested that both might stabilize the ferrous oxy ( $\mathrm{Fe}(\mathrm{II}) \mathrm{O}_{2}$ ) complex of the P450 to a greater extent than in WT BM3. To investigate further, we analysed reactivity of reduced WT and mutant NPG-bound heme domains with oxygen, using the substrate-bound species to enable complete reduction with sodium dithionite in all cases. For WT BM3 heme domain at $20^{\circ} \mathrm{C}$, mixing of the ferrous NPG-bound protein (in absence of excess dithionite) with aerobic oxygen results in negligible formation of a $\mathrm{Fe}(\mathrm{II}) \mathrm{O}_{2}$ complex, and the reoxidized (ferric) form is near-completely reformed within one second. The F261E heme domain behaved similarly to WT BM3. These data for WT BM3 heme domain are consistent with previous studies, which estimated a rate of conversion $\left(k_{\mathrm{ox}}\right)$ of the $\mathrm{Fe}(\mathrm{II}) \mathrm{O}_{2}$ complex to $\mathrm{Fe}(\mathrm{III})$ and superoxide at $0.22 \mathrm{~s}^{-1}$ at $20^{\circ} \mathrm{C}$ [42]. A more complete conversion to the $\mathrm{Fe}(\mathrm{II}) \mathrm{O}_{2}$ form is seen for WT heme domain at $-25^{\circ} \mathrm{C}$, with $k_{\mathrm{ox}}=0.1 \mathrm{~min}^{-1}\left(0.0017 \mathrm{~s}^{-1}\right)$ [43]. Ost et al. reported that $\mathrm{Fe}(\mathrm{II}) \mathrm{O}_{2}$ complexes of the $\mathrm{F} 393 \mathrm{~A} / \mathrm{H}$ BM3 heme domains were considerably stabilized compared to WT, decaying with a half-life of $\sim 30 \mathrm{~s}\left(k_{\mathrm{ox}} \sim 0.023 \mathrm{~s}^{-1}\right)$ at $15^{\circ} \mathrm{C}$ [19]. By comparison with the WT heme domain, the I401E and L86E mutants showed 
enhanced $\mathrm{Fe}(\mathrm{II}) \mathrm{O}_{2}$ complex stabilization. Spectral signatures of the NPG-bound $\mathrm{Fe}(\mathrm{II}) \mathrm{O}_{2}$ forms of these mutants are shown in Figure 5. For I401E at $20^{\circ} \mathrm{C}$, there is a spectral shift to $419 \mathrm{~nm}$ (with a shoulder at $442 \mathrm{~nm}$ ) at maximal population of the $\mathrm{Fe}(\mathrm{II}) \mathrm{O}_{2}$ species, prior to its collapsing back to the ferric Soret at $395 \mathrm{~nm}$ with a $k_{\mathrm{ox}}=0.0574 \mathrm{~s}^{-1}$ (half life $\sim 12.1 \mathrm{~s}$ ). For the L86E heme domain, the $\mathrm{Fe}(\mathrm{II}) \mathrm{O}_{2}$ absorbs maximally at $423 \mathrm{~nm}$, prior to decaying to the ferric form (again at $395 \mathrm{~nm}$ ) with a $k_{\mathrm{ox}}=0.1102 \mathrm{~s}^{-1}$ (half life $\sim 6.3 \mathrm{~s}$ ). To analyse the EPR properties of WT and mutant BM3 heme domains in their ferrous-oxy forms, samples were prepared as described in the Experimental section, and rapidly frozen. EPR revealed a complete absence of signals that could be assigned to a ferric superoxo form of the proteins, even in the cases of the I401E and L86E mutants where considerable formation of the oxy complex is seen spectrophotometrically (Figure 5). Minor signals $(<20 \%)$ assigned to the reoxidized (ferric) forms of the complexes were present. The absence of EPR signals for the ferrous-oxy forms is consistent with previous work on e.g. P450cam and the human aromatase (CYP19) P450 [44,45]. We conclude that the oxygen-bound WT BM3 and mutant heme domains are most likely in the ferric superoxo form, and are EPR silent as a consequence of the iron and superoxide being antiferromagnetically coupled.

\section{Steady-state turnover of mutant P450 BM3 enzymes}

To analyse catalytic properties of each flavocytochrome P450 BM3 mutant, steady-state analysis of fatty acid substrate-dependent NADPH oxidation was carried out. The substrates laurate and arachidonate were used. Data are summarized in Table 3. With the exception of L86E with laurate as substrate (where $k_{\text {cat }}$ is slightly higher than for WT BM3), all mutants had lower $k_{\text {cat }}$ values than WT BM3. For I401E, and despite a more rapid transfer of the first electron to the heme iron, overall catalytic rate is decreased $\sim 8$-fold with arachidonic acid. A likely explanation is that the elevated heme iron potential results in a decreased driving force for oxygen reduction by the ferrous heme iron, as suggested previously for F393A/H variants [19]. As expected from the high redox potential of the I401E heme iron and its ability to form the Fe(II)CO complex in absence of fatty acid substrate, the I401E flavocytochrome is a much more efficient NADPH oxidase in absence of fatty acids than is WT BM3. The rate of substrate-independent NADPH oxidation is $\sim 3.05 \mathrm{~s}^{-1}$ (I401E) compared to $\sim 0.03 \mathrm{~min}^{-1}$ for WT P450 BM3 in aerobic buffer. The comparable rates are $1.37 / 0.22 \mathrm{~s}^{-1}$ for the L86E/F261E mutants, respectively.

\section{Analysis of hydroxylated products formed from oxidation of lauric acid}

To verify that mutant flavocytochromes remained active in fatty acid oxidation, products were isolated from turnover reactions with lauric acid and identified by GC-MS. Lauric acid was converted completely to products for WT and all mutants. In all cases, there was evidence of the formation of $\omega-1, \omega-2$ and $\omega-3$ hydroxylaurate products, although the product ratios obtained varied between the enzymes. For WT BM3, the hydroxylated products were approximately equally distributed between $\omega-1, \omega-2$ and $\omega-3$ positions $(35 \%, 32 \%$ and $33 \%)$, consistent with previous work [e.g. 46]. The data for the L86E mutant showed a similar $\omega-1, \omega-2$ and $\omega-3$ product distribution to WT $(42 \%, 28 \%$ and 30\%), while F261E's profile was biased towards $\omega-3$ $(17 \%, 35 \%$ and $48 \%)$ and I401E's was towards $\omega-1(57 \%, 19 \%$ and $24 \%)$. Thus, all mutants were functional, but affected in regioselectivity of substrate oxidation, although not the positions of oxidation. Of particular note was the influence of I401E in favouring $\omega-1$ hydroxylation of laurate, since this mutation resides on the proximal side of the heme cofactor and cannot directly influence interactions with the substrate. Only monohydroxylated lauric acid products were observed in these assays.

\section{Structural studies on P450 BM3 mutants}

Crystal structures of the BM3 I401E, L86E and F261E heme domains indicated no covalent interaction with heme substituent (methyl or other) groups in all mutants, consistent with preceding data. The I401E/F261E mutant heme domains were crystallized in the substrate-free 
form, while the L86E heme domain structure could only be resolved for the substrate (NPG)bound form.

L86E heme domain: The NPG-bound L86E heme domain crystals diffracted to a resolution of 1.8 $\AA$. The L86E structure is nearly identical to the previously resolved WT heme domain NPG complex (PDB code 1JPZ) [47]. In contrast to the 1JPZ structure, the L86E NPG complex structure reveals partial occupancy of the distal water (modelled at 0.35 occupancy) in both active sites in the asymmetric unit. This suggests that NPG binding is not necessarily incompatible with retention of a distal water ligand to the heme iron and only partially drives conversion to the penta-coordinate state under the crystal conditions. In contrast to the relatively large structural changes induced by other point mutants in the heme vicinity (e.g. A264E) [15,32], the L86E mutation has little effect on heme domain structure, with the obvious exception of the immediate Glu86 environment. The Glu86 side chain is in hydrogen bonding contact with a heme propionate group. This, in turn, leads to a small reorganization in the orientation of His 100. The latter residue is now in direct polar contact with both Glu86 and a heme propionate (Figure 6). These novel interactions with heme peripheral substituent groups may explain the perturbation of the L86E heme iron redox potential.

I401E heme domain: The crystals of the I401E heme domain were obtained in absence of substrate and diffracted to $2.0 \AA$. The I401E structure is nearly identical to that of previously resolved substrate-free heme domain structures (e.g. 1BU7) [48]. The Glu401 indirectly hydrogen bonds via a water molecule to a heme propionate, while the direct environment of the Cys400 ligand appears unaltered (Figure 6). We again infer that new interactions affecting the propionate group may be key to modulating heme iron potential in this mutant. Previous studies of Phe393 variants of BM3 also revealed large shifts in heme potential, despite apparent lack of structural rearrangements in the vicinity of the cysteinate ligand from crystallographic analysis [34].

F261E heme domain: Crystals of F261E heme domain were obtained in absence of substrate and diffracted to $1.7 \AA$. In contrast to I401E crystals, the F261E crystal form is similar to that previously reported for the A264H heme domain (2IJ3), and which was also observed for the WT heme domain (2IJ2) [16]. The introduction of the F261E mutation results in the largest structural changes of the three mutants reported here. The Glu261 side chain occupies a space distinct from that of Phe261 in the WT protein, and is located further from the heme moiety. This not only leads to reorientation of Leu233, but also introduces novel water molecules close to the heme porphyrin plane (and corresponding to the Phe261 position in the WT structure). Small adaptations in the backbone positions of Cys156-Gly157 and Gln257-Ile259 are observed, likely due to the altered positions of Leu233/Glu261 and the introduction of the new waters (Figure 6). The structural rearrangement and introduction of water molecules in the heme vicinity for the F261E mutant may underlie the smaller modulation of heme iron potential compared to the I401E/L86E mutants (where novel heme propionate interactions are observed).

\section{Discussion}

Several mammalian CYP4 family P450s form a covalent linkage between a conserved glutamate residue and the 5-methyl substituent on the heme in a turnover-dependent manner [10-13]. The A264E mutation in P450 BM3 (mimicking the I helix glutamate in CYP4s) did not induce such heme linkage, despite similarities in catalytic characteristics between BM3 and the CYP4 fatty acid hydroxylases. BM3 has great biotechnological potential, and thus routes to improving its operational stability are of interest [6,49]. With this in mind, we identified new sites and generated BM3 mutants where engineered glutamates might enable interaction with the heme 5methyl group and lead to turnover-dependent covalent heme linkage. These were F261E (in the I helix), L86E (in the BC loop between B'/C helices) and I401E (immediately following the 
proximal heme ligand, Cys400) (Figure 6). However, none of these mutants gave significant levels of covalent heme linkage in either flavocytochrome or heme domain proteins. In the mutant heme domain crystal structures, none of the engineered glutamates are in van der Waals contact with the heme. This is not surprising, given their relative differences in polarity and in the absence of any cross-link between the heme group and the glutamate side chains. However, conformation(s) that would bring the glutamate carboxylates into direct contact with the heme methyl groups are related by minor movements to the crystal structures, and are thus likely easily accessible at room temperature. Indeed, our EPR studies indicate that conformations distinct from those seen in the crystals can be trapped at cryogenic temperatures at least for the F261E/L86E mutants, since in both these cases there is evidence for heme iron coordination by the introduced glutamates (Figure 3).

The absence of glutamate-heme methyl group ligation in the BM3 mutants may result from mechanistic differences between mammalian CYP4 and BM3 enzymes. In the former, it is postulated that production of compound $1\left(\mathrm{cpd} 1\right.$, a highly reactive ferryl-oxo $\left[\mathrm{Fe}^{\mathrm{IV}}=\mathrm{O}\right]$ porphyrin cation radical species considered as the active oxidant of $\mathrm{P} 450$ substrates) results in oxidation of the glutamate to produce a carboxylic radical with reduction of cpd1 to cpd2. This radical then abstracts a hydrogen atom from the heme 5-methyl group, forming a methylene radical which is then converted to a carbocation via intra-heme electron transfer. The carbocation may then be trapped by the glutamate carboxylate to form the ester linkage between protein and the heme methyl [14]. BM3 is a substantially more efficient enzyme than the CYP4s, with electrons delivered from its attached CPR domain, and between opposite CPR and P450 domains in a BM3 dimer [50]. Electron transfer rate between BM3 FMN and heme is $>200 \mathrm{~s}^{-1}$ at ambient temperature and overall turnover rate of the enzyme in steady-state is $>15,000 \mathrm{~min}^{-1}\left(250 \mathrm{~s}^{-1}\right)$ with arachidonate $[23,35]$. These values are 1-2 orders of magnitude greater than for the CYP4 enzymes. We speculate that cpd1 may be more transient in BM3 than in CYP4s, in addition to any considerations regarding non-optimal positioning of engineered glutamates in BM3 compared to CYP4s, where evolution has likely configured the relevant glutamates specifically for formation of a covalent bridge to the heme methyl.

Notwithstanding the inability of P450 BM3 to covalently link various engineered glutamates to the heme, profound effects are observed on the thermodynamic, substrate/ligand binding and catalytic features of the L86E, F261E and I401E mutants, as well as on stabilization of the ferrous-oxy forms of the L86E/I401E variants. Particularly notable is the substantially improved affinity of the L86E mutant for both laurate/arachidonate substrates, with $K_{\mathrm{d}}$ values at least an order of magnitude lower than for the WT BM3 (Table 1). This is consistent with the purification of the L86E heme domain in a partially HS state, likely indicating co-purification of the enzyme with some E. coli lipid bound. Previously, we co-purified the Bacillus subtilis P450 BioI (CYP107H1) protein from E. coli bound to palmitic acid, which is a predominant lipid in E. coli and a tight binding substrate for both P450 BioI and BM3 [51]. This enhanced fatty acid affinity was also manifest in an improved $K_{\mathrm{m}}$ for lauric acid with the L86E flavocytochrome, accompanied by an 21 .5-fold improvement in $k_{\text {cat }}$ for this substrate. The structural origins of this improved affinity are uncertain, since the NPG-bound L86E heme domain structure is highly similar to the WT NPG complex. However, the substrate-free L86E heme domain was not readily crystallized and substrate-free crystals obtained diffracted poorly, suggesting altered conformational states and/or equilibria between these states in the L86E mutant. Enhanced substrate affinity and altered structural conformations were also observed previously for the BM3 A264E mutant [32,33]. The I401E heme domain also displayed higher affinity for lauric and arachidonic acids compared to WT BM3, with the $K_{\mathrm{d}}$ for laurate improved $\sim 7$-fold (Table 1). However, despite substrate $K_{\mathrm{m}}$ values comparable (or lower) to those for WT P450 BM3, the $k_{\text {cat }}$ values were considerably lower. This is likely as a consequence of over-stabilization of the 
ferrous-oxy complex and retardation of subsequent electron transfer and oxygen activation steps $[52]$.

Potentiometry revealed positive shifts in heme iron potential (relative to WT) in all mutants, and for both substrate-free and arachidonate-bound heme domains (Table 2). The potential of $-219 \pm$ $4 \mathrm{mV}$ for the substrate-free I401E heme domain is the most positive yet reported for a substratefree BM3 mutant, and $>100 \mathrm{mV}$ more positive than for the substrate-free F393A/H mutants (312/-332 $\mathrm{mV}$, respectively). Arachidonate binding increased the heme iron potentials of the F393A/H heme domains to $-151 /-176 \mathrm{mV}$, respectively, similar to the value for the arachidonatebound I401E heme domain [19]. The mutant substrate-free heme potentials are closer to that for the catalytically relevant form of the FMN cofactor (the oxidized/semiquinone couple at $-240 \mathrm{mV}$ [41]) than is that for WT BM3. All mutant heme potentials are more positive than $-240 \mathrm{mV}$ in their substrate-bound forms. This is consistent with the higher rates of substrate-independent NADPH oxidation in the mutants. Table 2 reveals a reasonable correlation of FMN-to-heme electron transfer rate with heme iron potential and the proportion of HS heme for the substratefree WT/mutant BM3 enzymes. In the substrate-bound enzymes the heme potentials are further elevated, and FMN-to-heme electron transfer is accelerated. The correlation between rate and heme potential/HS proportion is not absolute for the arachidonate-bound WT and mutants, although heme reduction rate is fastest for I401E $\left(k_{\lim }=473 \mathrm{~s}^{-1}\right)$, consistent with its potential being $119 \mathrm{mV}$ more positive than arachidonate-bound WT BM3, and the L86E and F261E mutants (that have a lower $k_{\lim }$ than WT) also show lower arachidonate-induced conversion to HS heme iron. Crystal structures revealed new interactions between heme propionate and Glu401 in I401E BM3, and between Glu86 and heme propionate/His100 in L86E BM3 (Figure 6). Considerable structural rearrangements also occurred in the F261E heme domain, including introduction of novel waters in the heme vicinity that may affect its electrostatic environment. Mutant heme domain structures were also obtained only in the substrate-bound form (L86E) or in an infrequently observed substrate-free conformation (F261E), pointing to structural perturbations that could also impact on efficiency of FMN-to-heme electron transfer.

Wong and co-workers noted that a L358P mutation immediately following the cysteinate ligand increased ethane/propane oxidation in P450cam [53]. They also showed that the BM3 I401P mutant had a substrate-free heme potential of $-303 \mathrm{mV}(142 \mathrm{mV}$ more positive than WT) and that coupling of NADPH oxidation to lauric acid hydroxylation was similar in the I401P mutant (53\%) to WT BM3 (52\%) [21]. The I401P flavocytochrome also displayed an 16-fold increase in substrate-independent NADPH oxidation. Our data for the I401E mutant are consistent with these findings, although the substrate-free I401E potential $(-219 \mathrm{mV})$ is $\sim 84 \mathrm{mV}$ more positive than for the I401P mutant. The I401E, L86E and F261E enzymes all remain functional in fatty acid oxidation, but with notable alterations in regioselectivity of laurate oxidation. The $\omega-1$ hydroxylaurate product is favoured in the F261E mutant, with a substantial shift towards $\omega-3$ laurate hydroxylation in the I401E mutant. The coupling of NADPH oxidation to lauric acid oxidation was $\geq 40 \%$ for all mutant enzymes analysed in this study.

The perturbed potentials of the I401E/L86E heme domains resulted in stabilization of the ferrousoxy form (compared to WT), enabling the species to be readily characterized optically (Figure 5) and for samples to be frozen for EPR analysis. However, EPR showed no signal attributable to a ferric superoxo form, even in the I401E mutant in which lifetime of the oxy complex is substantially increased. It is considered that electronic redistribution of this oxy complex occurs in the P450 catalytic cycle to favour a ferric superoxo species, thus withdrawing electron density from the heme iron to favour a second electron transfer that leads to a transient ferric peroxo intermediate. The ferrous-oxy complexes of P450cam and human aromatase are also EPR silent, although Mossbauer and resonance Raman analysis of the P450cam complex provide compelling 
data for ferric heme iron and oxygen in the superoxide form [54,55]. Thus, we conclude that the WT and mutant P450s BM3 analysed here are likely in the ferric superoxo form, with their ferric heme iron and bound superoxide antiferromagnetically coupled to produce an EPR silent species.

\section{Conclusions}

The I401E, L86E and F261E glutamate replacement mutants near the heme 5-methyl group in P450 BM3 induced perturbations to substrate/ligand affinity (including improved $K_{\mathrm{d}}$ values for laurate and arachidonate in I401E/L86E mutants) and kinetics of substrate oxidation. Conformational flexibility of mutant heme domains was apparent both from evidence of some coordination of heme iron by Glu264/Glu86 in low temperature EPR studies of the relevant mutants, and from the distinctive conformational states in which the three mutants were crystallized. The mutations induced heme potential changes in all mutants, with considerable positive shifts in the $\mathrm{Fe}(\mathrm{III}) / \mathrm{Fe}(\mathrm{II})$ potential of the $\mathrm{L} 86 \mathrm{E} / \mathrm{I} 401 \mathrm{E}$ mutants resulting in greater stability of the ferrous-oxy species, and an $\sim 2$-fold increase in FMN-to-heme electron transfer rate in the substrate-bound I401E flavocyochrome. All mutants retained lauric acid hydroxylase activity, with altered regioselectivity in the F261E ( $\omega-1$ oxidation favoured) and I401E $(\omega-3$ oxidation favoured) mutants. Although there was no evidence of covalent linkage of heme 5methyl to the mutant proteins, similar strategies of introducing charged residues in the heme environment may prove useful for stabilization of heme oxy complexes, and for alteration of substrate selectivity and regioselectivity of substrate oxidation in BM3 and other P450s.

\section{Acknowledgements}

The authors acknowledge the financial support of the UK Biotechnology and Biological Sciences Research Council (BBSRC, grants BB/F00252/1 and BB/F00883X1) for this research. We are also grateful to Prof. W. Ewen Smith (University of Strathclyde, UK) for access to resonance Raman facilities.

\section{References}

1) Denisov, I.G., Makris, T.M., Sligar, S.G. and Schlichting, I. (2005) Structure and chemistry of cytochrome P450. Chem. Rev. 105, 2253-2277.

2) Guengerich, F.P., Wu, Z.L. and Bartleson, C.J. (2005). Function of human cytochrome P450s: characterization of the orphans. Biochem. Biophys. Res. Commun. 338, 465-469.

3) Cupp-Vickery, J.R., Han, O., Hutchinson, C.R. and Poulos, T.L. (1996). Substrate-assisted catalysis in cytochrome P450eryF. Nat. Struct. Biol. 3, 632-637.

4) Lawson, R.J., Leys, D., Sutcliffe, M.J., Kemp, C.A., Cheesman, M.R., Smith, S.J., Clarkson, J., Smith, W.E., Haq, I., Perkins, J.B. and Munro, A.W. (2004). Thermodynamic and biophysical characerterization of cytochrome P450 BioI from Bacillus subtilis. Biochemistry 43, 1241012426.

5) Poulos, T.L. (2003). The past and present of P450cam structural biology. Biochem. Biophys. Res. Commun. 312, 35-39.

6) Munro, A.W. Leys, D.G., McLean, K.J., Marshall, K.R., Ost, T.W., Daff, S., Miles, C.S., Chapman, S.K., Lysek, D.A., Moser, C.C., Page, C.C. and Dutton, P.L. (2002). P450 BM3: The very model of a modern flavocytochrome. Trends Biochem. Sci. 27, 250-257.

7) Perera, R., Sono, M., Sigman, J.A., Pfister, T.D., Lu, Y. and Dawson, J.H. (2003). Neutral thiol as a proximal ligand to ferrous heme iron: implications for heme proteins that lose cysteine thiolate ligation on reduction. Proc. Natl. Acad. Sci. USA 100, 3641-3646.

8) Munro, A.W., Girvan, H.M. and McLean, K.J. (2007). Cytochrome P450-redox partner fusion enzymes. Biochim. Biophys. Acta 1770, 345-359. 
9) Munro, A.W., Girvan, H.M. and McLean, K.J. (2007) Variations on a (t)heme - novel mechanisms, redox partners and catalytic functions in the cytochrome P450 superfamily. Nat. Prod. Rep. 24, 585-609.

10) Colas, C. and Ortiz de Montellano, P.R. (2003). Autocatalytic radical reactions in physiological prosthetic heme modification. Chem. Rev. 103, 2305-2332.

11) Henne, K.R., Kunze, K.L., Zheng, Y.M., Christmas, P., Soberman, R.J. and Rettie, A.E. (2001). Covalent linkage of prosthetic heme to CYP4 family enzymes. Biochemistry 40, 1292512931.

12) LeBrun, L.A., Hoch, U. and Ortiz de Montellano, P.R. (2002). Autocatalytic mechanism and consequences of covalent heme attachment in the cytochrome P4504A family. J. Biol. Chem. 277, 12755-12761.

13) Hoch, U. and Ortiz de Montellano, P.R. (2001). Covalently linked heme in cytochrome P4504A fatty acid hydroxylases. J. Biol. Chem. 276, 11339-11346.

14) Limburg, J., LeBrun, L.A. and Ortiz de Montellano, P.R. (2005). The P450cam G248E mutant covalently binds its prosthetic heme group. Biochemistry 44, 4091-4099.

15) Girvan, H.M., Marshall, K.R., Lawson, R.J., Leys, D., Joyce, M.G., Clarkson, J., Smith, W.E., Cheesman, M.R. and Munro, A.W. (2004). Flavocytochrome P450 BM3 mutant A264E undergoes substrate-dependent formation of a novel heme iron ligand set. J. Biol. Chem. 279, 23274-23286.

16) Girvan, H.M., Seward, H.E., Toogood, H.S., Cheesman, M.R., Leys, D. and Munro, A.W. (2007). Structural and spectroscopic characterization of P450 BM3 mutants with unprecedented $\mathrm{P} 450$ heme iron ligand sets. New heme ligation states influence conformational equilibria in P450 BM3. J. Biol. Chem. 282, 564-572.

17) Girvan, H.M., Toogood, H.S., Littleford, R.E., Seward, H.E., Smith, W.E., Ekanem, I.S., Leys, D., Cheesman, M.R. and Munro, A.W. (2009). Novel haem co-ordination variants of flavocytochrome P450 BM3. Biochem. J. 417, 65-76.

18) Yeom, H., Sligar, S.G., Li, H., Poulos, T.L. and Fulco, A.J. (1995). The role of Thr268 in oxygen activation of cytochrome P450 BM3. Biochemistry 34, 14733-14740.

19) Ost, T.W., Miles, C.S., Munro, A.W., Murdoch, J., Reid, G.A. and Chapman, S.K. (2001). Phenylalanine 393 exerts thermodynamic control over the heme of flavocytochrome P450 BM3. Biochemistry 40, 13421-13429.

20) Ravichandran, K.G., Boddupalli, S.S., Hasemann, C.A., Peterson, J.A. and Deisenhofer, J. (1993). Crystal structure of hemoprotein domain of P450BM-3, a prototype for microsomal P450s. Science 261, 731-736.

21) Whitehouse, J.C., Bell, S.G., Yang, W., Yorke, J.A., Blanford, C.F., Strong, A.J.F., Morse, E.J., Bartlam, M., Rao, Z. and Wong, L.-L. (2009). A highly active single-mutation variant of P450 BM3 (CYP102A1). ChemBioChem 10, 1654-1656.

22) Miles, J.S., Munro, A.W., Rospendowski, B.N., Smith, W.E., McKnight, J. and Thomson, A.J. (1992). Domains of the catalytically self-sufficient cytochrome P-450 BM-3. Genetic construction, overexpression, purification and spectroscopic characterization. Biochem. J. 288, 503-509.

23) Noble, M.A., Miles, C.S., Chapman, S.K., Lysek, D.A., MacKay, A.C., Reid, G.A., Hanzlik, R.P. and Munro, A.W. (1999). Roles of key active-site residues in flavocytochrome P450 BM3. Biochem. J. 339, 371-379.

24) Omura, T and Sato, R. (1964). The carbon monoxide-binding pigment of liver microsome. I. Evidence for its hemoprotein nature. J. Biol. Chem. 239, 2370-2378.

25) McLean, K.J., Cheesman, M.R., Rivers, S.L., Richmond, A., Leys, D., Chapman, S.K., Reid, G.A., Price, N.C., Kelly, S.M., Clarkson, J., Smith, W.E. and Munro, A.W. (2002). Expression, purification and spectroscopic characterization of the cytochrome P450 CYP121 from Mycobacterium tuberculosis. J. Inorg. Biochem. 91, 527-541. 
26) Dutton, P.L. (1978). Redox potentiometry: Determination of midpoint potentials of oxidation-reduction components of biological electron-transfer systems. Methods Enzymol. 54, 411-435.

27) Daff, S.N., Chapman, S.K., Turner, K.L., Holt, R.A., Govindaraj, S., Poulos, T.L. and Munro, A.W. (1997). Redox control of the catalytic cycle of flavocytochrome P-450 BM3. Biochemistry 36, 13816-13823.

28) Kabsch, W. (1988). Automatic indexing of rotation diffraction patterns. J. Appl. Cryst. 21, 67-71.

29) Collaborative Computational Project, Number 4. (1994) The CCP4 Suite: Programs for protein crystallography. Acta Cryst. D50, 760-763.

30) Murshudov, G.N. Vagin, A.A. \& Dodson, E.J. (1997). Refinement of macromolecular structures by the maximum-likelihood method. Acta Cryst. D53, 240-255.

31) Emsley P, Cowtan K (2004) Coot: model-building tools for molecular graphics. Acta Cryst. D60: $2126-2132$.

32) Joyce, M.G., Girvan, H.M., Munro, A.W. and Leys, D. (2004). A single mutation in cytochrome P450 BM3 induces the conformational rearrangement seen upon substrate binding in the wild-type enzyme. J. Biol. Chem. 279, 23287-23293.

33) Li, H.Y. and Poulos, T.L. (1997). The structure of the cytochrome P450BM-3 haem domain complexed with the fatty acid substrate, palmitoleic acid. Nature Struct. Biol. 4, 140-146.

34) Perera, R., Sono, M., Sigman, J.A., Pfister, T.D., Lu, Y. and Dawson, J.H. (2003). Neutral thiol as a proximal ligand to ferrous heme iron: implications for heme proteins that lose cysteine thiolate ligation on reduction. Proc. Natl. Acad. Sci. USA 100, 3641-3646.

35) Dunford, A.J., McLean, K.J., Sabri, M., Seward, H.E., Heyes, D.J., Scrutton, N.S. and Munro, A.W. (2007). Rapid P450 heme iron reduction by laser photoexcitation of Mycobacterium tuberculosis CYP121 and CYP51B1. Analysis of CO complexation reactions and reversibility of the P450/P420 equilibrium. J. Biol. Chem. 282, 24816-24824.

36) $\mathrm{Hu}$, S., Smith, K.M. and Spiro, T.G. (1996). Assignment of protoheme resonance Raman spectrum by heme labelling in myoglobin. J. Am. Chem. Soc. 118, 12638-12646.

37) Smith, S.J., Munro, A.W. and Smith, W.E. (2003). Resonance Raman scattering of cytochrome P450 BM3 and the effect of imidazole inhibitors. Biopolymers 70, 620-627.

38) Ost, T.W.B., Munro, A.W., Mowat, C.G., Taylor, P.R., Pesseguiero, A., Fulco, A.J., Cho, A.K., Cheesman, M.R., Walkinshaw, M.D. and Chapman, S.K. (2001). Structural and spectroscopic analysis of the F393H mutant of flavocytochrome P450 BM3. Biochemistry 40, 13430-13438.

39) Munro A.W., Daff, S., Coggins, J.R., Lindsay, J.G. and Chapman, S.K. (1996). Probing electron transfer in flavocytochrome P450 BM3 and its component domains. Eur. J. Biochem. 239, 403-409.

40) Girvan, H.M., Heyes, D.J., Scrutton, N.S. and Munro, A.W. (2007). Laser photoexcitation of $\mathrm{NAD}(\mathrm{P}) \mathrm{H}$ induces reduction of P450 BM3 heme domain on the microsecond time scale. J. Am. Chem. Soc. 129, 6647-6653.

41) Hanley, S.C. and Daff, S. (2004). The unusual redox properties of flavocytochrome P450 BM3 flavodoxin domain. Biochem. Biophys. Res. Commun. 325, 1418-1423.

42) Sevrioukova, I.F. and Peterson, J.A. (1995). Reaction of carbon monoxide and molecular oxygen with P450terp (CYP108) and P450BM-3 (CYP102). Arch. Biochem. Biophys. 317, 397404.

43) Bec, N., Anzenbacher, P., Anzenbacherova, E., Gorren, A.C.F., Munro, A.W. and Lange, R. (1999). Spectral properties of the oxyferrous complex of the heme domain of cytochrome P450 BM-3 (CYP102). Biochem. Biophys. Res. Commun. 266, 187-189.

44) Davydov, R., Makris, T.M., Kofman, V., Werst, D.E., Sligar, S.G. and Hoffman, B.M. (2001). Hydroxylation of camphor by reduced oxy-cytochrome P450cam: mechanistic 
implications of EPR and ENDOR studies of catalytic intermediates in native and mutant enzyme. J. Am. Chem. Soc. 123, 1403-1415.

45) Gantt, S., Denisov, I.G., Grinkova, Y.V. and Sligar, S.G. (2009). The critical iron-oxygen intermediate in human aromatase. Biochem. Biophys. Res. Commun. 387, 169-173.

46) Boddupalli, S.S., Pramanik, B.C., Slaughter, C.A., Estabrook, R.W. and Peterson, J.A. (1992). Fatty acid monooxygenation by P450BM-3: product identification and proposed mechanisms for the sequential hydroxylation reactions. Arch. Biochem. Biophys. 292, 20-28.

47) Haines, D.C., Tomchick, D.R., Machius, M. and Peterson J.A. (2001). Pivotal role of water in the mechanism of P450BM-3. Biochemistry 40, 13456-13465.

48) Sevrioukova, I.F., Li, H., Zhang, H., Peterson, J.A., and Poulos, T.L. (1999). Structure of a cytochrome P450-redox partner electron-transfer complex. Proc. Natl. Acad. Sci. USA 96, 1863-1868.

49) Ost, T.W., Miles, C.S., Murdoch, J., Cheung, Y., Reid, G.A., Chapman, S.K. and Munro A.W. (2000). Rational re-design of the substrate binding site of flavocytochrome P450 BM3. FEBS Lett. 486, 173-177.

50) Neeli, R., Girvan, H.M., Lawrence, A., Warren, M.J., Leys, D., Scrutton, N.S. and Munro, A.W. (2005). The dimeric form of flavocytochrome P450 BM3 is catalytically functional as a fatty acid hydroxylase. FEBS Lett. 579, 5582-5588.

51) Lawson, R.J., Leys, D., Sutcliffe, M.J., Kemp, C.A., Cheesman, M.R., Smith, S.J., Smith, W.E., Haq, I., Perkins, J.B. and Munro, A.W. (2004). Thermodynamic and biophysical characterization of cytochrome P450 BioI from Bacillus subtilis. Biochemistry 43, 12410-12426.

52) Ost, T.W., Clark, J., Mowat, C.G., Miles, C.S., Walkinshaw, M.D., Reid, G.A., Chapman, S.K. and Daff, S. (2003). Oxygen activation and electron transfer in flavocytochrome P450 BM3. J. Am. Chem. Soc. 125, 15010-15020.

53) Xu, F., Bell, S.G. Lednik, J., Insley, A., Rao, Z. and Wong, L.-L. (2005). The heme monooxygenase cytochrome P450cam can be engineered to oxidize ethane to ethanol. Angew. Chem. Int. Ed. 44, 4029-4032.

54) Sharrock, M., Debrunner, P.G., Schulz, C., Lipscomb, J.D., Marshall, V. and Gunsalus, I.C. (1976). Cytochrome P450cam and its complexes. Mossbauer parameters of the heme iron. Biochim. Biophys. Acta 420, 8-26.

55) Bangcharoenpaurpong, O., Rizos, A.K., Champion, P.M., Jollie, D. and Sligar, S.G. (1986). Resonance Raman detection of bound dioxygen in cytochrome P-450cam. J. Biol. Chem. 261, 8089-8092. 


\section{Tables}

Table 1. Dissociation constants for substrate and inhibitor ligand binding to WT and mutant P450 BM3 heme domains. Dissociation constants $\left(K_{\mathrm{d}}\right.$ values) were determined from optical titrations, as described in the Experimental section. Plots of ligand-induced optical change versus cyanide concentration were sigmoidal in the case of the F261E and I401E heme domains (indicated by an asterisk) and were fitted using a sigmoidal (Hill) function to obtain an apparent $K_{\mathrm{d}}\left(K_{\mathrm{H}}\right)$; whereas corresponding plots for WT and the L86E heme domains were hyperbolic and were fitted using a standard hyperbolic function.

\begin{tabular}{|c|c|c|c|c|}
\cline { 2 - 5 } \multicolumn{1}{c|}{} & \multicolumn{3}{c|}{ Dissociation constant $\left(\boldsymbol{K}_{\mathbf{d}}\right)$} \\
\hline Substrate/ligand & WT & F261E & I401E & L86E \\
\hline Arachidonic acid & $0.55 \pm 0.05 \mu \mathrm{M}$ & $0.90 \pm 0.06 \mu \mathrm{M}$ & $0.26 \pm 0.04 \mu \mathrm{M}$ & $0.02 \pm 0.01 \mu \mathrm{M}$ \\
\hline Lauric acid & $89.0 \pm 15.0 \mu \mathrm{M}$ & $>1 \mathrm{mM}$ & $11.9 \pm 1.1 \mu \mathrm{M}$ & $1.0 \pm 0.15 \mu \mathrm{M}$ \\
\hline 4-PIM & $0.85 \pm 0.45 \mu \mathrm{M}$ & $3.94 \pm 0.20 \mu \mathrm{M}$ & $0.10 \pm 0.03 \mu \mathrm{M}$ & $13.1 \pm 0.5 \mu \mathrm{M}$ \\
\hline $\mathrm{NaCN}$ & $1.68 \pm 0.06 \mathrm{mM}$ & $49.0 \pm 0.3 \mathrm{mM}^{*}$ & $41.2 \pm 0.4 \mathrm{mM}$ & $14.7 \pm 0.5 \mathrm{mM}$ \\
\hline
\end{tabular}

Table 2. Heme iron reduction potentials and $\mathrm{Fe}(\mathrm{II}) \mathrm{CO}$ complex formation rates for WT and mutant $\mathbf{P 4 5 0}$ BM3 heme domains. Heme iron $\left(\mathrm{Fe}^{3+} / \mathrm{Fe}^{2+}\right)$ reduction potentials for substrate-free and arachidonate-bound P450 BM3 heme domains were determined under identical conditions by spectroelectrochemical titration, as described in the Experimental section. Values are reported relative to the normal hydrogen electrode (NHE). Stopped-flow rates $\left(k_{\text {lim }}\right)$ of $\mathrm{Fe}(\mathrm{II}) \mathrm{CO}$ complex formation (reflecting FMN-to-heme electron transfer rate) in presence and absence of arachidonate were also measured as described in the Experimental section and are accurate to $\leq$ $5 \%$ in each case. Values in parentheses are estimates of the proportion of the heme iron in the ferric high-spin state in the WT or mutant P450 BM3 enzyme used for the experiment.

\begin{tabular}{|l|c|c|c|c|}
\cline { 2 - 5 } \multicolumn{1}{c|}{} & \multicolumn{2}{c|}{$\begin{array}{c}\text { Redox Potential versus } \\
\text { NHE }(\mathbf{m V})\end{array}$} & \multicolumn{2}{c|}{$\begin{array}{c}\text { Fe(II)CO complex } \\
\text { formation rate }\left(\boldsymbol{k}_{\text {lim }}, \mathbf{s}^{-1}\right)\end{array}$} \\
\cline { 2 - 5 } \multicolumn{1}{c|}{} & $\begin{array}{c}\text { Substrate- } \\
\text { free }\end{array}$ & $\begin{array}{c}\text { Arachidonate } \\
- \text { bound }\end{array}$ & $\begin{array}{c}\text { Substrate- } \\
\text { free }\end{array}$ & $\begin{array}{c}\text { Arachidonate- } \\
\text { bound }\end{array}$ \\
\hline WT & $-395 \pm 5$ & $-285 \pm 3$ & $5(<5 \%)$ & $250(>90 \%)$ \\
\hline F261E & $-367 \pm 8$ & $-197 \pm 6$ & $27(<5 \%)$ & $58(70 \%)$ \\
\hline I401E & $-219 \pm 4$ & $-166 \pm 7$ & $60(<5 \%)$ & $473(>90 \%)$ \\
\hline L86E & $-307 \pm 5$ & $-176 \pm 4$ & $102(10 \%)$ & $213(65 \%)$ \\
\hline
\end{tabular}


Table 3. Steady-state kinetic parameters for WT and mutant P450 BM3 enzymes. Data were collected as described in the Experimental section. *The apparent arachidonate-dependent NADPH oxidation rate was linear with respect to fatty acid concentration for the F261E mutant, and a second order rate constant is provided for this mutant/fatty acid combination.

\begin{tabular}{|c|c|c|c|c|}
\cline { 2 - 5 } \multicolumn{1}{c|}{} & \multicolumn{2}{c|}{ Laurate } & \multicolumn{2}{c|}{ Arachidonate } \\
\cline { 2 - 5 } \multicolumn{1}{c|}{} & $k_{\text {cat }}\left(\mathrm{min}^{-1}\right)$ & $K_{\mathrm{M}}(\mu \mathrm{M})$ & $k_{\text {cat }}\left(\mathrm{min}^{-1}\right)$ & $K_{\mathrm{M}}(\mu \mathrm{M})$ \\
\hline Wild-type & $2770 \pm 120$ & $87.4 \pm 8.1$ & $16400 \pm 185$ & $5.1 \pm 0.4$ \\
\hline L86E & $4010 \pm 480$ & $31.6 \pm 10.2$ & $7538 \pm 891$ & $11.5 \pm 3.4$ \\
\hline F261E & $608 \pm 58$ & $1563 \pm 213$ & $15.8 \pm 2.0 \min ^{-1}$ & $\mu \mathrm{M}^{-1 *}$ \\
\hline I401E & $926 \pm 98$ & $23.0 \pm 1.5$ & $1970 \pm 178$ & $4.7 \pm 1.5$ \\
\hline
\end{tabular}


Structure and catalysis in P450 BM3 mutants

\section{Figure Legends}

\section{Figure 1. Arachidonate binding to mutant P450 BM3 heme domains}

Panel A shows selected UV-visible absorption spectra for binding of arachidonate to I401E BM3 heme domain $(2.75 \mu \mathrm{M})$. The starting spectrum, prior to substrate addition, is shown as a dotted line and the final one containing near-saturating arachidonate $(12.5 \mu \mathrm{M})$ as a thick, solid line. Intermediate spectra containing $1 \mu \mathrm{M}, 2 \mu \mathrm{M}, 3.5 \mu \mathrm{M}$ and $5 \mu \mathrm{M}$ arachidonate are shown in thin, solid lines. The Soret shift is from $418 \mathrm{~nm}$ to $395 \mathrm{~nm}$. The inset shows the arachidonate-induced optical shift $\left(\Delta \mathrm{A}_{378}-\Delta \mathrm{A}_{412} \mathrm{~nm}\right)$ data fitted to Equation $1\left(K_{\mathrm{d}}=259 \pm 40 \mathrm{nM}\right)$. Panel B shows the degree of spectral shift upon saturation of each mutant and WT heme domain with arachidonate. WT is shown as a thick, solid line; I401E as a thin, solid line; F261E as a dashed line; and L86E as a dotted line.

\section{Figure 2. Binding of ligands to the F261E mutant P450 BM3 heme domain}

Shown as a thin black line is F261E $(8 \mu \mathrm{M})$ in the oxidized state in the absence of any exogenous ligands. The 4-PIM-bound form (14 $\mu \mathrm{M}$ ligand) is shown as a dashed line. The CN-bound form $(90 \mathrm{mM})$ is shown as a bold, dashed-dotted line. The reduced $\left(\mathrm{Fe}^{2+}\right)$ form is shown as a dotted line, with the $\mathrm{Fe}^{2+}-\mathrm{CO}$ complex as a thick black line. $\mathrm{CN}$ has a very high apparent $K_{\mathrm{d}}(\sim 49 \mathrm{mM})$ and there is an incomplete Soret shift to a typical final maximum $(\sim 440 \mathrm{~nm})$ even at the high CN concentration used here.

Figure 3. X-band EPR spectra of WT and mutant heme domains of P450 BM3 EPR spectra were collected at $10 \mathrm{~K}$, as described in the Experimental section, and the spectra for substrate-free forms of WT, L86E, F261E and I401E heme domains are overlaid. The g-values for each protein at 2.42, 2.26 and 1.92/1.93 are consistent with LS thiolate-coordinated ferric heme iron. The additional species observed for the F261E $\left(g_{x}=2.63 / 2.52\right.$ and $\left.g_{z}=1.90 / 1.84\right)$ and $\mathrm{L} 86 \mathrm{E}\left(\mathrm{g}_{\mathrm{x}}=2.58 / 2.53 / 2.48\right.$ and $\left.\mathrm{g}_{\mathrm{z}}=1.90 / 1.87\right)$ likely indicates altered distal coordination, and may result from a proportion of molecules having Glu261/Glu86 as the distal heme iron ligand.

\section{Figure 4. Redox potentiometric analysis of I401E P450 BM3 heme domain}

Panel A shows UV-visible spectra accompanying the redox titration of substrate-free I401E heme domain $(7 \mu \mathrm{M})$. Shown as a thick solid line is the starting (oxidized) spectrum with its Soret maximum at $418 \mathrm{~nm}$. Titration with the reductant sodium dithionite resulted in heme spectral shifts, with the Soret maximum blue shifted. The final spectrum (showing fully reduced I401E heme domain, and corresponding to a potential of $-333 \mathrm{mV}$ vs. the normal hydrogen electrode, NHE) is shown as a dashed line, with its Soret maximum at $409 \mathrm{~nm}$. A number of intermediate spectra are shown as thin solid lines. Panel B shows the percentage of reduced heme iron (from spectral data at the relevant heme absorption maxima) plotted against the applied potential, and with data fitted using the Nernst function to generate the midpoint $\mathrm{Fe}^{3+} / \mathrm{Fe}^{2+}$ reduction potentials detailed in Table 2. In solid circles are data for substrate-free WT BM3 heme domain; open circles are for arachidonate-bound WT heme domain; solid triangles are for substrate-free I401E heme domain; and open triangles are for arachidonate-bound I401E heme domain.

Figure 5. Spectral features of the oxyferrous complexes of the I401E and L86E heme domains of P450 BM3. Panel A shows the spectral features of the dithionite-reduced ferrous (thick solid line), oxygenated $\mathrm{Fe}(\mathrm{II}) \mathrm{O}_{2}$ (thin solid line) and reoxidized ferric (dashed line) forms of the I401E heme domain in complex with NPG. Absorption maxima are at $410 \mathrm{~nm}, 419 \mathrm{~nm}$ and $395 \mathrm{~nm}$, respectively. Panel B shows the same spectral set for the NPG-bound L86E heme domain, with absorption maxima at $411 \mathrm{~nm}, 423 \mathrm{~nm}$ and $396 \mathrm{~nm}$. Spectra were collected following stopped-flow mixing of anaerobic, reduced P450s with oxygen-saturated buffer, with the first spectrum collected 1.28 milliseconds after the mixing event. The spectra shown for the 
$\mathrm{Fe}(\mathrm{II}) \mathrm{O}_{2}$ species of the I401E/L86E mutants were recorded after $11.5 \mathrm{~ms} / 6.5 \mathrm{~ms}$, respectively, and those for the reoxidized forms after 200 seconds.

Figure 6. Structural analysis of BM3 heme domains. Panel A shows the positions of mutated residues (blue) and Ala264 relative to the heme cofactor and its 5-methyl group (shown as a red sphere). A black asterisk in each of panels A-D further identifies the heme 5-methyl group. In panels B-D, the WT (green) and mutant structural elements (red) are shown for the heme, NPG substrate (in case of L86E, panel B), relevant amino acids and the oxygens of water molecules. $\mathrm{Red} / \mathrm{green}$ coloured dashed lines identify altered interactions with heme propionate in the L86E and I401E mutants. Panel B shows the L86E heme domain with NPG substrate at the top. A water molecule is apparently present on the heme iron in $\sim 35 \%$ of the molecules. The Glu 86 residue is in hydrogen bonding contact with a heme propionate, and this structural rearrangement also impacts on His 100 such that its side chain now also makes polar contact with the propionate and Glu86. Panel C shows the F261E heme domain, with the Glu261 side chain occupying a distinctive position from that of Phe261 in the WT structure, and further from the heme. There is marked change in orientation of Leu233 and in the backbone positions of the Cys156-Gly157 and Gln257-Ile259 peptides. Novel water molecules (labelled W1 and W2) are also introduced in the vicinity of the heme. Panel D shows the I401E heme domain, with Glu401 hydrogen bonded to one of the heme propionates via a water molecule. 


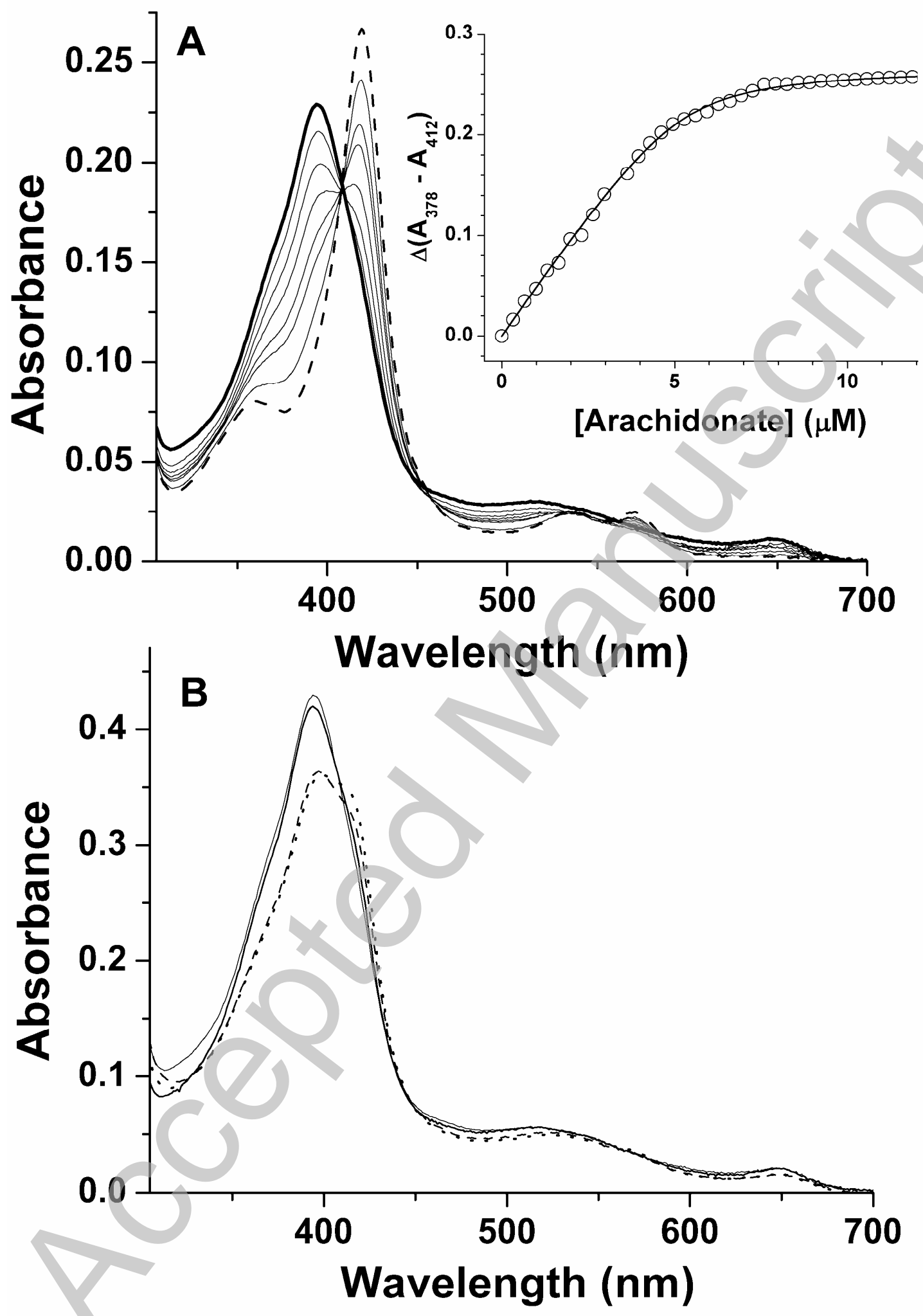

Figure 1 


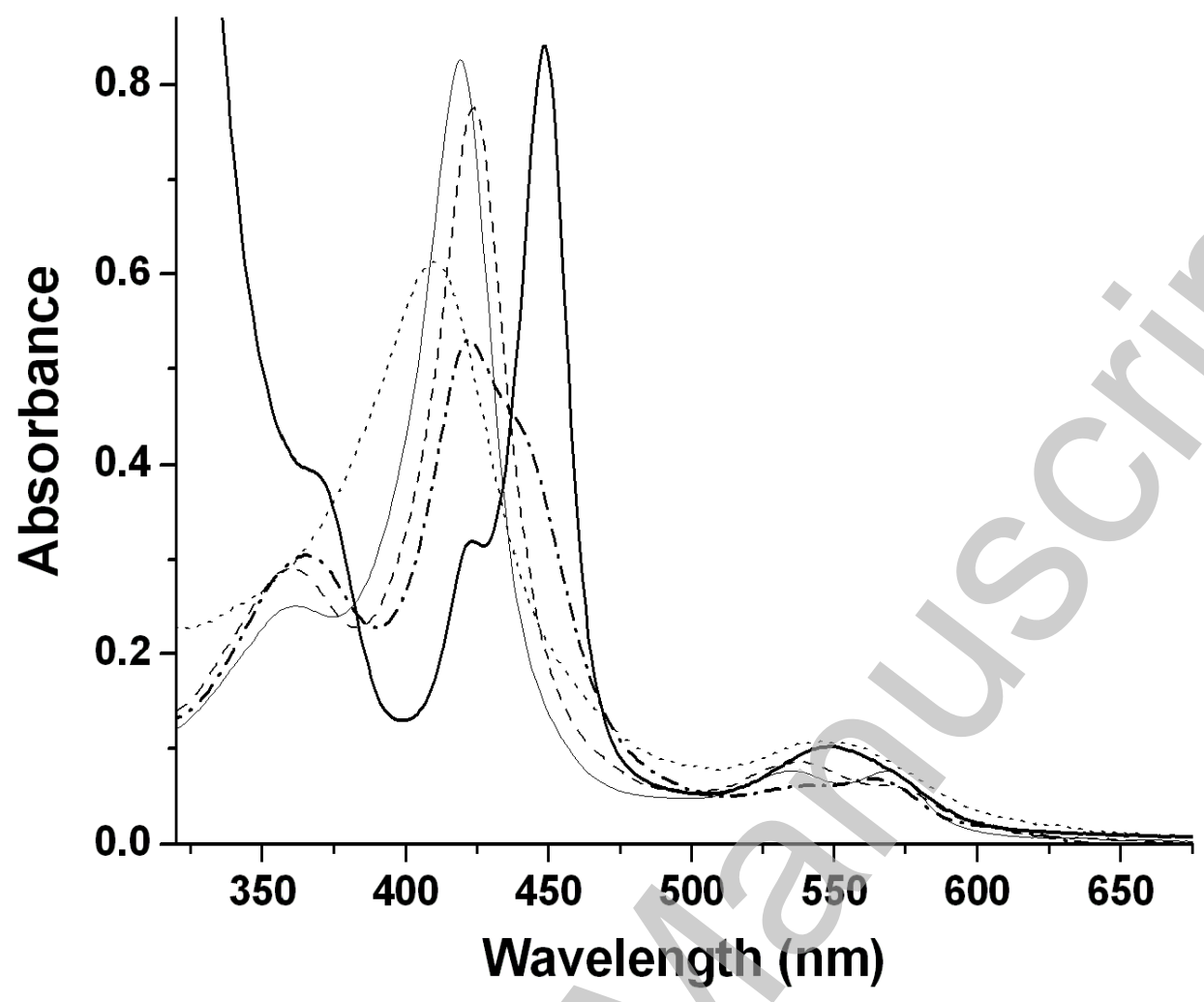

Figure 2 


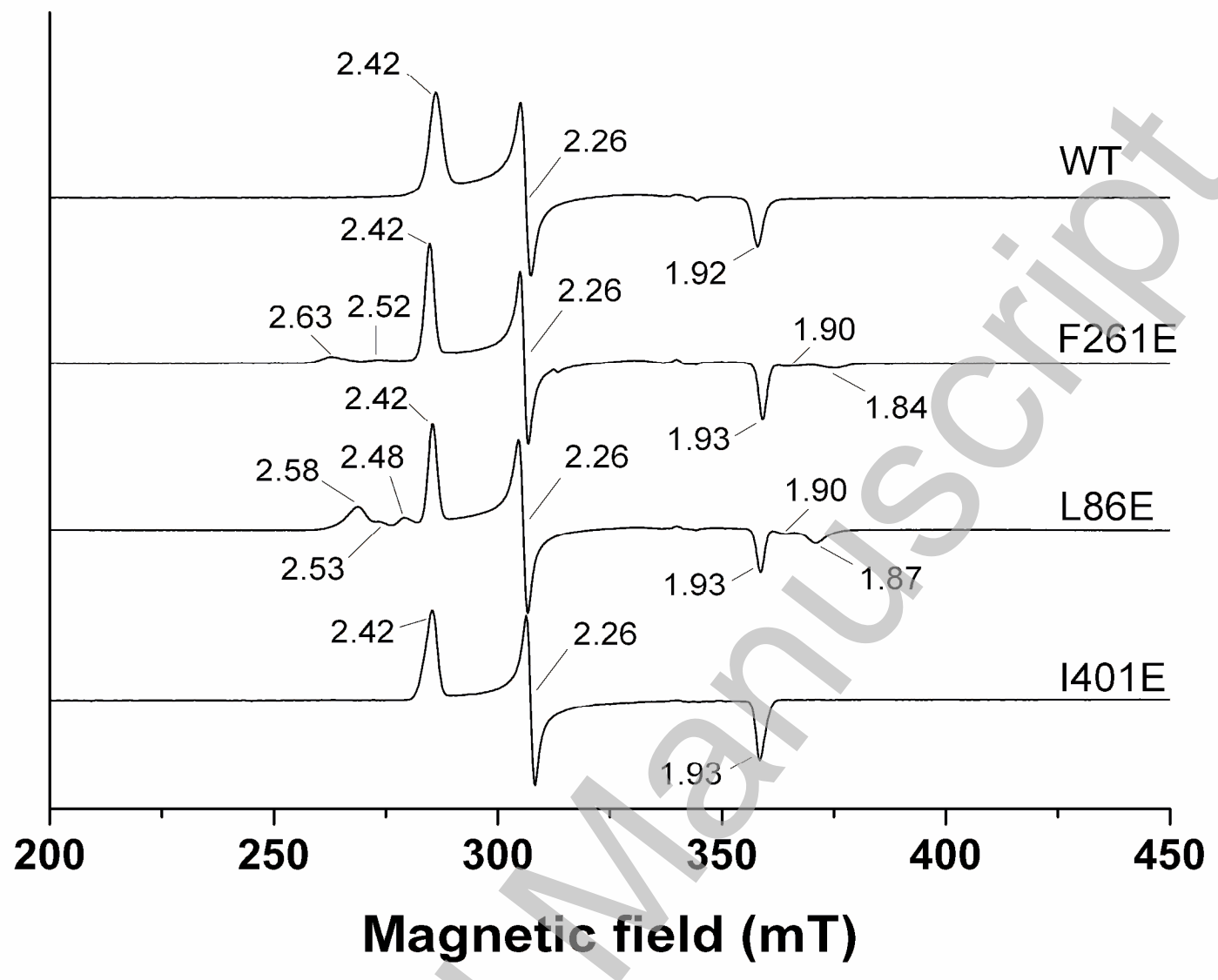

Figure 3 

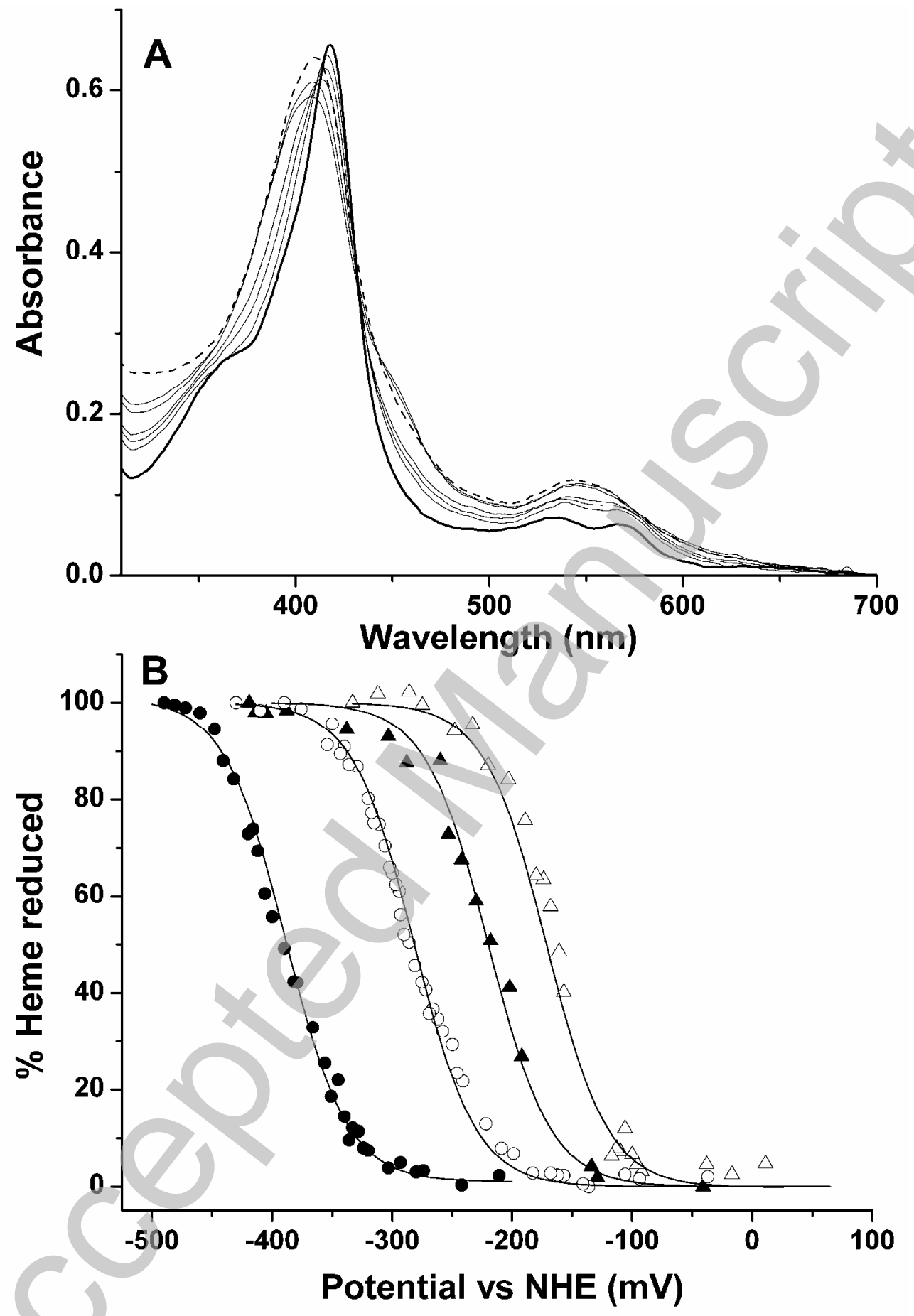

Figure 4 

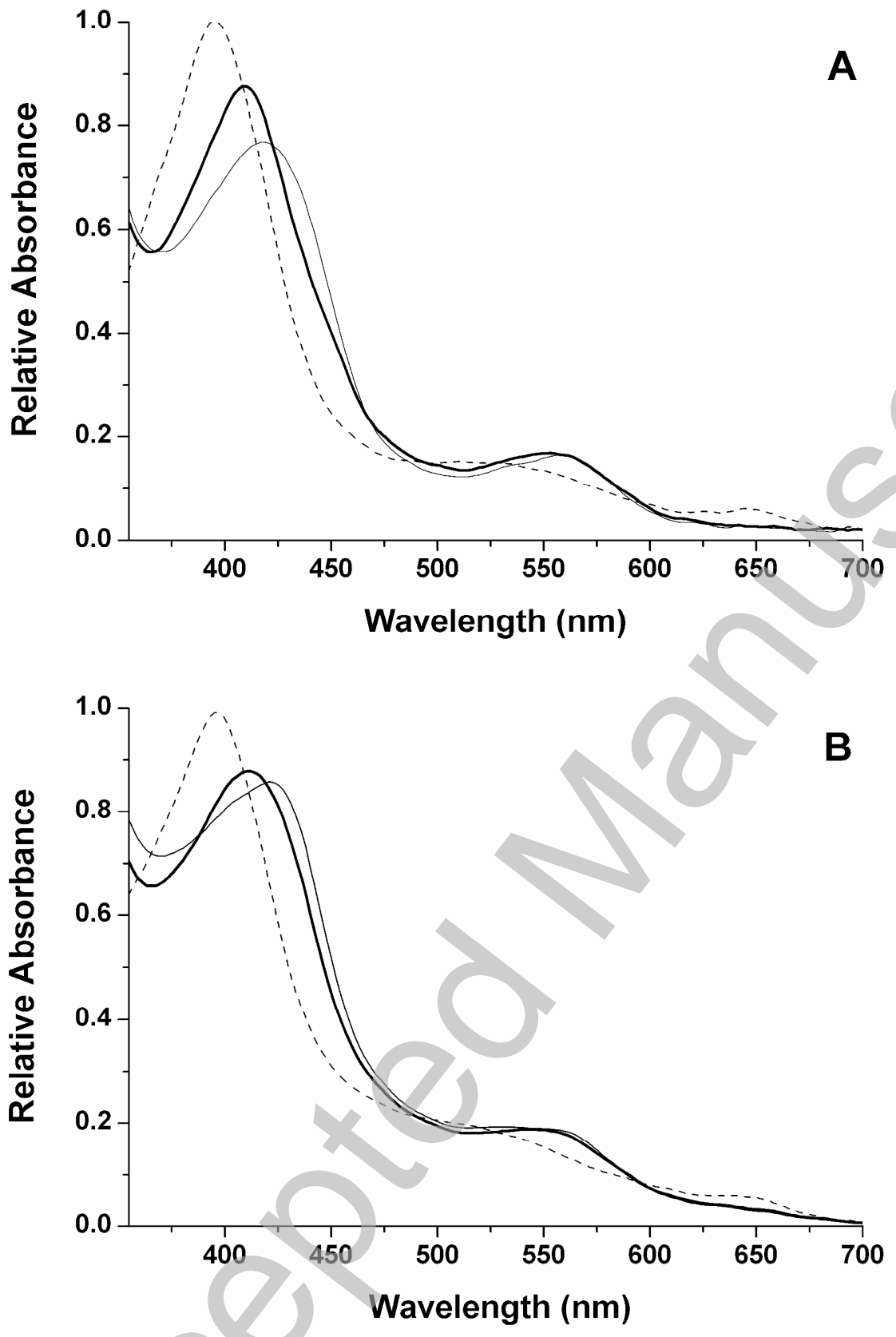

Figure 5 

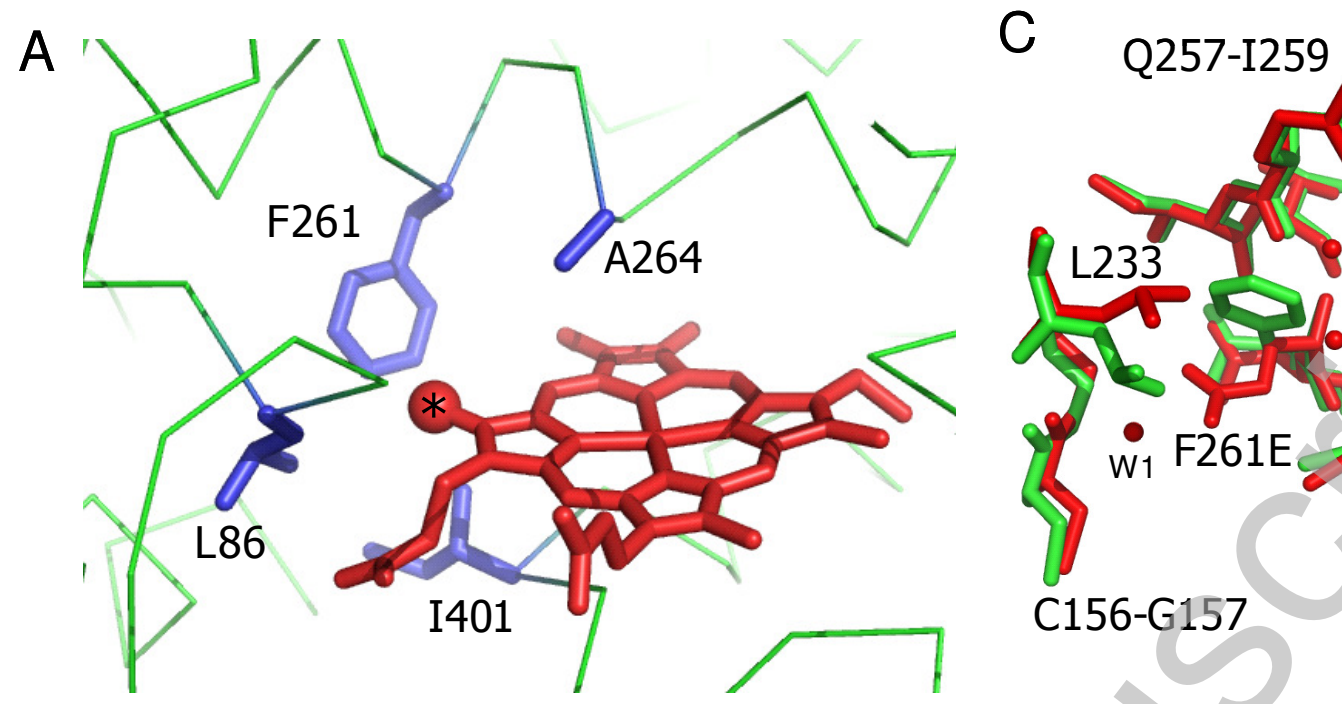

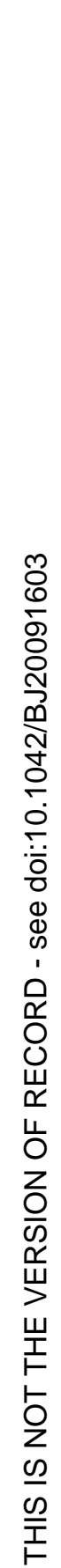

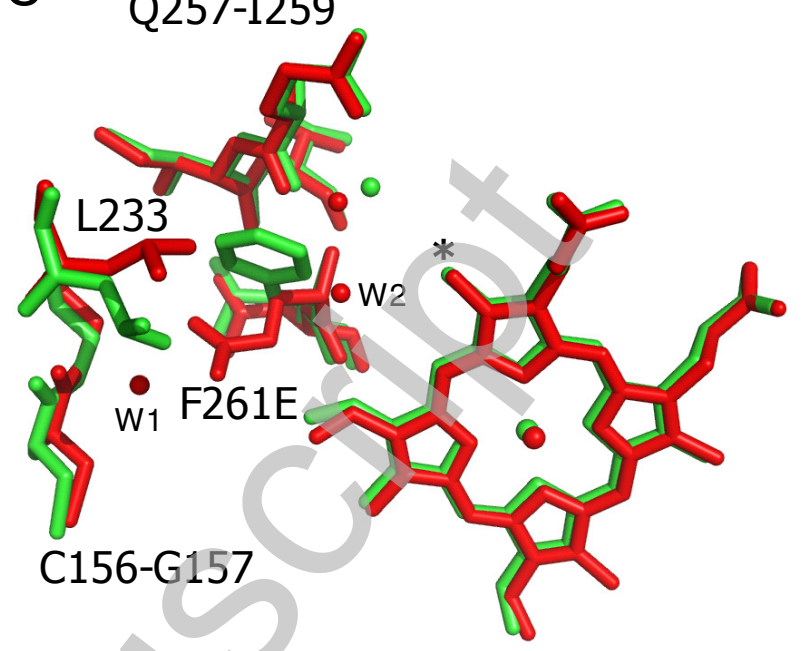

B
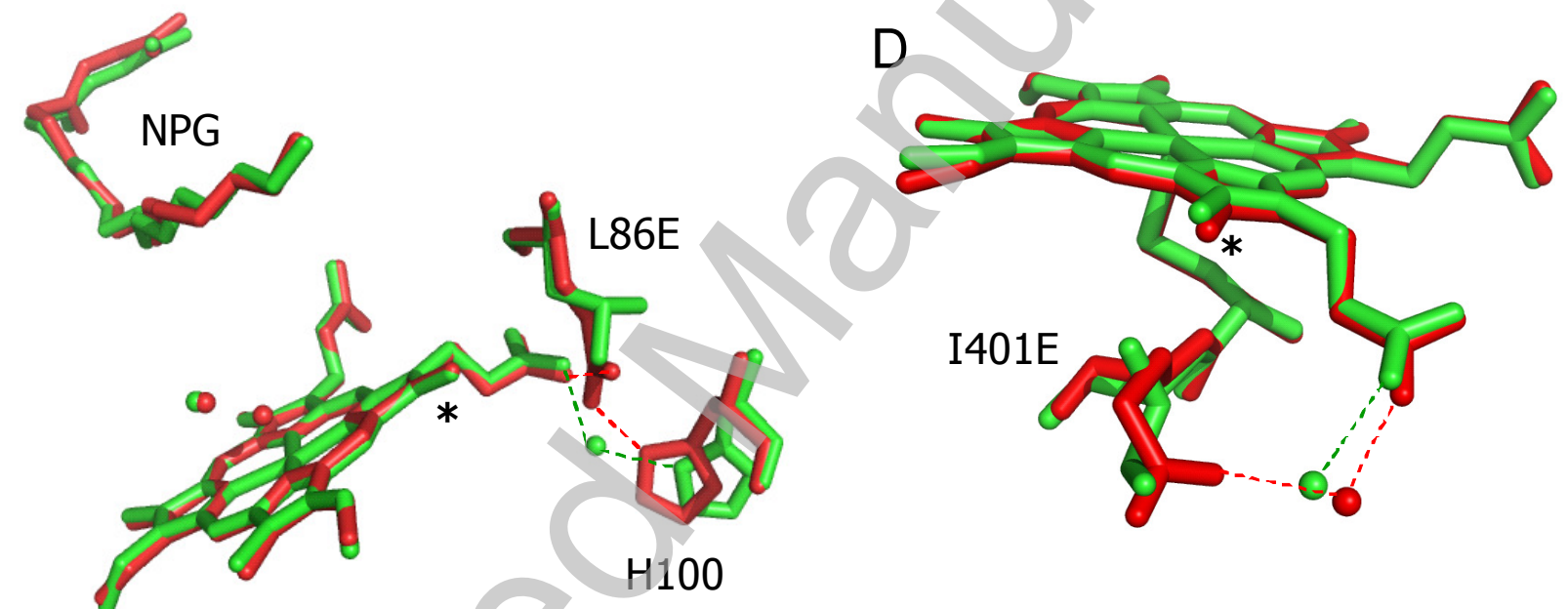

Figure 6 